\title{
Causality in quantum optics and entanglement of Minkowski vacuum
}

\author{
Anatoly Svidzinsky $\odot,{ }^{1}$ Arash Azizi $\odot,{ }^{1}$ Jonathan S. Ben-Benjamin, ${ }^{1}$ Marlan O. Scully, ${ }^{1,2,3}$ and William Unruh ${ }^{1,4}$ \\ ${ }^{1}$ Texas A\&M University, College Station, Texas 77843, USA \\ ${ }^{2}$ Baylor University, Waco, Texas 76798, USA \\ ${ }^{3}$ Princeton University, Princeton, New Jersey 08544, USA \\ ${ }^{4}$ University of British Columbia, Vancouver, Canada V6T $1 Z 1$
}

(Received 22 April 2020; revised 16 December 2020; accepted 8 February 2021; published 1 March 2021)

\begin{abstract}
Emission of photons by atoms can occur into modes which extend into a region causally disconnected with the emitter. For example, a uniformly accelerated ground-state atom emits a photon into the Unruh-Minkowski mode which is exponentially larger in the causally disconnected region. This makes an impression that photon emission is acausal. Here we show that conventional quantum optical analysis yields that a detector atom will not detect the emitted photon in the region noncausally connected with the emitter. However, joint excitation probability of atoms in the causally disconnected regions can be correlated due to entanglement of Minkowski vacuum and be much larger than the product of independent excitation probabilities. Moreover, atoms uniformly accelerated in the same Rindler wedge cannot become simultaneously excited without changing the state of the field, that is, the Unruh-Minkowski photon emitted by one atom cannot be absorbed by the other atom. We discuss examples demonstrating interesting features of Minkowski vacuum entanglement.
\end{abstract}

DOI: 10.1103/PhysRevResearch.3.013202

\section{INTRODUCTION}

The principle of relativistic causality that signals should propagate no faster than the speed of light is of fundamental importance for the foundations of physics. If a signal could be transmitted with superluminal velocity, then there would exist a Lorentz frame in which the cause (switching on of the signal source) would be later than the effect (arrival of the signal). And one could then have a purely logical contradiction of the type in which the effect could occur before its cause. In the literature it has been argued that quantum theory has issues with the principle of relativistic causality (see, e.g., Refs. [1-6]). In particular, the problems with causality beyond the rotatingwave approximation are known in the Glauber-Kelley-Kleiner photodetection theory [7-10]. Several ways to resolve the issue have been suggested (see, e.g., Refs. [11-13]).

One can envision the problem with causality if we consider a mode into which a photon is emitted by a ground-state atom uniformly accelerated in Minkowski vacuum. The process of photon emission is accompanied by the atom's excitation. This is known as the Unruh effect [14] (or the Fulling-DaviesUnruh effect in full [14-16]). Interpretation of the effect depends on a choice of the reference frame.

A noninertial observer having a proper constant acceleration $a$, i.e., a Rindler observer [17], sees that space is filled with thermal photons with Unruh temperature $T_{U}$ proportional

Published by the American Physical Society under the terms of the Creative Commons Attribution 4.0 International license. Further distribution of this work must maintain attribution to the author(s) and the published article's title, journal citation, and DOI. to the acceleration [14],

$$
T_{U}=\frac{\hbar a}{2 \pi k_{B} c} .
$$

From the perspective of the accelerated observer, the groundstate atoms accelerated through Minkowski vacuum, will be promoted to the excited state by the absorption of the Rindler particles (Unruh effect) [14]. However, an inertial observer interprets the absorption of a Rindler particle as the emission of a Minkowski particle [18], which is known as acceleration radiation. From the perspective of the inertial observer, the energy is gained from the atom's kinetic energy. A similar mechanism yields excitation of an atom freely falling in a gravitational field [19] or an atom uniformly moving through an optical cavity [20] or a fixed atom in the presence of an accelerated mirror [21] .

For simplicity, in this paper we consider either dimension $1+1$ or dimension $3+1$ but restrict photons to have wavevector $\mathbf{k}$ parallel to the $z$ axis. We assume that the field is scalar and obeys the one-dimensional wave equation,

$$
\frac{1}{c^{2}} \frac{\partial^{2} \phi}{\partial t^{2}}-\frac{\partial^{2} \phi}{\partial z^{2}}=0
$$

The inner product of two field modes $\phi_{1}(t, z)$ and $\phi_{2}(t, z)$ obeying Eq. (2) is defined as the Klein-Gordon inner product which is a generalization of the Wronksian,

$$
\begin{aligned}
\left\langle\phi_{1}, \phi_{2}\right\rangle & =\frac{i}{2} \int_{-\infty}^{\infty}\left(\phi_{1}^{*} \pi_{2}^{*}-\pi_{1} \phi_{2}\right) d z \\
& =\frac{i}{2 c} \int_{-\infty}^{\infty}\left(\phi_{1}^{*} \frac{\partial \phi_{2}}{\partial t}-\frac{\partial \phi_{1}^{*}}{\partial t} \phi_{2}\right) d z
\end{aligned}
$$




\section{Minkowski space}

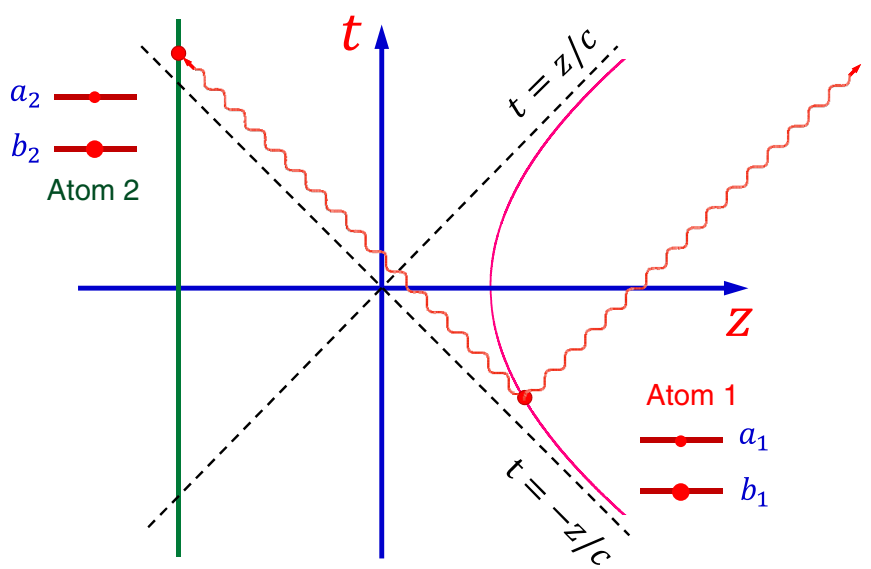

FIG. 1. Atom 1 accelerates from $-\infty$ to $+\infty$ along the hyperbolic trajectory. Unruh acceleration radiation from atom 1 is shown as wavy lines which is absorbed by the stationary detector atom 2 .

where $\pi=c \partial L / \partial \dot{\phi}=(1 / c) \partial \phi^{*} / \partial t$ is the conjugate momentum. The inner product is independent of time and has the following properties:

$$
\left\langle\phi_{1}^{*}, \phi_{1}^{*}\right\rangle=-\left\langle\phi_{1}, \phi_{1}\right\rangle, \quad\left\langle\phi_{1}, \phi_{1}^{*}\right\rangle=\left\langle\phi_{1}^{*}, \phi_{1}\right\rangle=0 .
$$

The modes,

$$
\begin{aligned}
\phi_{1 v} & =\sqrt{\frac{a}{v c}}(\mp z-c t)^{i(v c / a)} \theta(\mp z-c t), \\
\phi_{2 v} & =\sqrt{\frac{a}{v c}}(c t \pm z)^{-i(v c / a)} \theta(c t \pm z)
\end{aligned}
$$

are solutions of the wave equation (2) and are known as Rindler modes. Here $v$ is the photon frequency in the Rindler space [see Eq. (11) below]. For $v>0$ modes (5) and (6) have positive norm [defined in Eq. (3)] which, however, diverges as in the case of plane waves. The upper and the lower signs in Eqs. (5) and (6) correspond to the left- and right-propagating photons, respectively. The mode functions (5) and (6) are nonzero in half of the $t-z$ plane and form a complete basis set.

The coordinate transformation $t, z \rightarrow \bar{t}, \bar{z}$,

$$
\begin{aligned}
& t=\frac{1}{\alpha} e^{\alpha \bar{z} / c} \sinh (\alpha \bar{t}), \\
& z=\frac{c}{\alpha} e^{\alpha \bar{z} / c} \cosh (\alpha \bar{t}),
\end{aligned}
$$

where $\alpha=a / c>0$ is a constant, converts the Minkowski space-time line element $d s^{2}=c^{2} d t^{2}-d z^{2}$ in the right Rindler wedge $z>c|t|$ to the Rindler line element [17],

$$
d s^{2}=e^{2 \alpha \bar{z} / c}\left(c^{2} d \bar{t}^{2}-d \bar{z}^{2}\right) .
$$

An atom moving along the trajectory $\bar{z}=$ const in the $\bar{t}-\bar{z}$ (Rindler) space has constant proper acceleration $\bar{a}=a e^{-\alpha \bar{z} / c}$ and proper time $\tau=e^{\alpha \bar{z} / c} \bar{t}$. For $\bar{z}=0$ the atom moves along the trajectory (see Fig. 1),

$$
t(\tau)=\frac{c}{a} \sinh \left(\frac{a \tau}{c}\right), \quad z(\tau)=\frac{c^{2}}{a} \cosh \left(\frac{a \tau}{c}\right),
$$

in the Minkowski space. The normal modes of scalar photons (5) and (6) in the Rindler space take the same form as the usual positive norm plane waves in the Minkowski metric, that is

$$
\phi_{1 v}(\bar{t}, \bar{z}) \propto e^{-i v(\bar{t} \pm \bar{z} / c)}, \quad \phi_{2 v}(\bar{t}, \bar{z}) \propto e^{-i v(\bar{t} \pm \bar{z} / c)},
$$

where $v$ is the photon angular frequency in the Rindler space. However, modes (11) are a mixture of positive and negative frequency modes with respect to the physical Minkowski space-time. Therefore, the vacuum state of these modes is not Minkowski vacuum but rather Rindler vacuum.

Taking superposition of the Rindler-mode functions (5) and (6), one can construct the so-called Unruh-Minkowski modes [18],

$$
\begin{aligned}
F_{1 v}(t, z) & =\frac{|t \pm z / c|^{i(v c / a)}}{\sqrt{2 \frac{v c}{a} \sinh \left(\frac{\pi v c}{a}\right)}} \begin{cases}e^{-(\pi v c) / 2 a}, & t \pm z / c>0, \\
e^{(\pi v c) / 2 a}, & t \pm z / c<0,\end{cases} \\
& =\frac{e^{-(\pi v c) / 2 a}}{\sqrt{2 \frac{v c}{a} \sinh \left(\frac{\pi v c}{a}\right)}}(t \pm z / c-i \lambda)^{i(\nu c / a),}
\end{aligned}
$$

and

$$
\begin{aligned}
F_{2 v}(t, z) & =\frac{|t \pm z / c|^{-i(v c / a)}}{\sqrt{2 \frac{v c}{a} \sinh \left(\frac{\pi v c}{a}\right)}} \begin{cases}e^{(\pi v c / 2 a)}, & t \pm z / c>0, \\
e^{-(\pi v c / 2 a)}, & t \pm z / c<0,\end{cases} \\
& =\frac{e^{(\pi v c / 2 a)}}{\sqrt{2 \frac{v c}{a} \sinh \left(\frac{\pi v c}{a}\right)}}(t \pm z / c-i \lambda)^{-i(v c / a)} .
\end{aligned}
$$

Here $\lambda=0+, v>0$, and the \pm sign corresponds to left- and right-propagating photons, respectively. The mode functions (12) and (13) differ by changing $v \rightarrow-v$. They depend only on the combinations $t \pm z / c$, that is, describing waves traveling with the speed of light $c$. In Fig. 2 we plot the absolute value and the phase of $F_{1 v}(t, z)$ as a function of $t \pm z / c$ for $v c / a=4$. The mode function $F_{1 v}(t, z)$ is exponentially small for $t \pm z / c>0$, whereas the mode function phase logarithmically diverges when $|t \pm z / c| \rightarrow 0$.

The Unruh-Minkowski modes (12) and (13) are solutions of Eq. (2). They form a complete set and have positive norm and, thus, are associated with the photon annihilation operators $\hat{a}_{1 v}$ and $\hat{a}_{2 v}$. The negative-norm modes are the complex conjugates of Eqs. (12) and (13) and correspond to the photon creation operators $\hat{a}_{1 v}^{\dagger}$ and $\hat{a}_{2 v}^{\dagger}$. The vacuum state for the Unruh-Minkowski photons is usual Minkowski vacuum $\left|0_{M}\right\rangle$, that is, $\hat{a}_{1 v}\left|0_{M}\right\rangle=0, \hat{a}_{2 v}\left|0_{M}\right\rangle=0$ for all $v$.

Operators for the Rindler photons $\hat{b}_{v}$ and for the UnruhMinkowski photons $\hat{a}_{v}$ are related by the Bogoliubov-like transformation (see Appendix A),

$$
\hat{b}_{1 v}=\frac{\hat{a}_{1 v}+e^{-\pi c v / a} \hat{a}_{2 v}^{\dagger}}{\sqrt{1-e^{-2 \pi c v / a}}}, \quad \hat{b}_{2 v}=\frac{\hat{a}_{2 v}+e^{-\pi c v / a} \hat{a}_{1 v}^{\dagger}}{\sqrt{1-e^{-2 \pi c v / a}}},
$$

which yields

$$
\hat{b}_{1 v}\left|0_{M}\right\rangle=\frac{\hat{a}_{2 v}^{\dagger}\left|0_{M}\right\rangle}{\sqrt{e^{2 \pi c v / a}-1}}, \quad \hat{b}_{2 v}\left|0_{M}\right\rangle=\frac{\hat{a}_{1 \nu}^{\dagger}\left|0_{M}\right\rangle}{\sqrt{e^{2 \pi c v / a}-1}},
$$




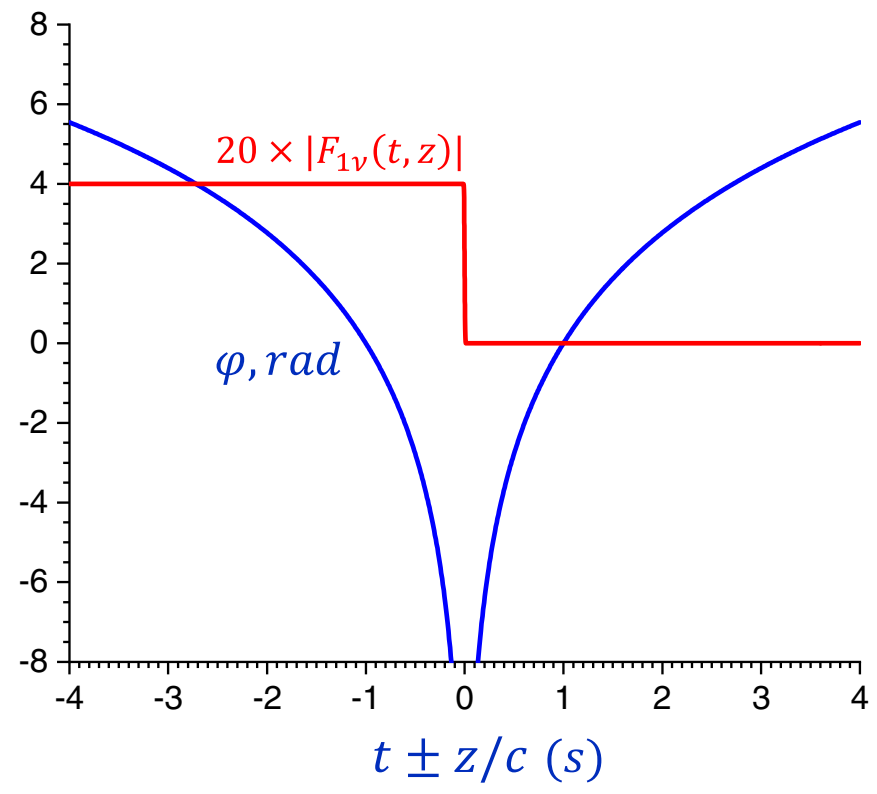

FIG. 2. The absolute value and the phase $\varphi$ of $F_{1 v}(t, z)$ given by Eq. (12) as a function of $t \pm z / c$ for $v c / a=4 .\left|F_{1 v}(t, z)\right|$ is multiplied by 20 for a better visualization.

and, therefore,

$$
\left\langle 0_{M}\left|\hat{b}_{1 v}^{\dagger} \hat{b}_{1 v}\right| 0_{M}\right\rangle=\left\langle 0_{M}\left|\hat{b}_{2 v}^{\dagger} \hat{b}_{2 v}\right| 0_{M}\right\rangle=\frac{1}{e^{2 \pi c v / a}-1} .
$$

That is a uniformly accelerated observer in Minkowski vacuum sees the presence of Rindler photons with the average mode occupation number given by the thermal Planck factor with Unruh temperature (1). Equation (15) shows that creation of the Unruh-Minkowski photons (13) and (12) out of Minkowski vacuum $\left|0_{M}\right\rangle$ can be viewed as annihilation of the Rindler photons (5) and (6), respectively.

A ground-state atom with transition frequency $\omega$ moving in the right Rindler wedge with acceleration $a$ (see Fig. 1) emits the left-propagating photon into the Unruh-Minkowski mode $F_{1 \omega}$ and the right-propagating photon into the mode $F_{2 \omega}$ [18]. In Appendix B, we obtain this result by performing calculations using the plane-wave modes as a basis set. The mode functions $F_{1 \omega}$ and $F_{2 \omega}$ are nonzero in a region noncausally connected with the emitting atom, namely, in the left Rindler wedge $z<-c|t|$. Moreover, they are in a factor $e^{-\pi c \omega / a}$ smaller in the causally connected region than outside that region. This makes an impression that photon emission by the accelerated atom is acausal and a detector atom 2 placed in the noncausally connected region can become excited by absorbing the photon emitted by the accelerated atom.

However, according to Eq. (15), emission of such UnruhMinkowski photons into the acausal region can be viewed as annihilation of the Rindler photons having the mode function that vanishes in the acausal region. Thus, from this perspective, there is no problem with causality.

Similar issues arise for the Fermi problem [11] where a stationary atom 1 with transition frequency $\omega$ spontaneously emits a photon which is then detected by atom 2 . In particular, the photon can be emitted into the right-propagating plane- wave mode,

$$
f_{\omega}(t, z)=\frac{1}{\sqrt{2 \omega}} e^{-i \omega(t-z / c)},
$$

which spreads into the noncausally connected region to the left of emitting atom 1. This makes an impression that such right-propagating photon can excite detector atom 2 located to the left of emitting atom 1.

In this paper we investigate the issues of causality and vacuum entanglement in the framework of conventional quantum optical analysis. In particular, we show that evolution of a detector atom is independent of the emitter if they are located in the causally disconnected regions. We also discuss interesting properties of photon emission by uniformly accelerated atoms. For example, we show that a photon emitted by a uniformly accelerated atom can not be absorbed by another atom accelerated in the same direction but can be absorbed by an atom accelerated in the opposite direction.

In Minkowski vacuum, the number of Rindler photons in the left and the right Rindler wedges is correlated. Since uniformly accelerated ground-state atoms absorb Rindler photons, they can be used as sensors to test correlations between the number of Rindler photons localized in different wedges. Joint probability that two ground-state atoms become excited and the field remains in Minkowski vacuum contain information about the Rindler photon number correlations. In Sec. IV we show that because of such correlations, the joint excitation probability can be exponentially larger than the product of independent excitation probabilities for the two atoms if they move in opposite Rindler wedges. In contrast, if the atoms move in the same wedge the joint excitation probability of atoms, provided the field remains in Minkowski vacuum, is equal to zero.

\section{EVOLUTION OPERATOR FOR THE DETECTOR-EMITTER SYSTEM}

We consider a pair of two-level ( $a$ and $b$ ) atoms with transition angular frequencies $\omega_{1}$ and $\omega_{2}$. Atom 2 serves as a detector which tests the field produced by atom 1 . The interaction between the detector atom and the field can be suddenly turned on and turned off at time $t$ or change with time adiabatically. Time-dependent coupling allows us to test the state of the field at a particular moment of time, however, it can also make the detector atom excited.

The interaction Hamiltonian between the atoms and the field is

$$
\hat{V}(t)=\hat{V}_{1}(t)+\hat{V}_{2}(t),
$$

where

$$
\begin{gathered}
\hat{V}_{1}(t)=g\left(\hat{\sigma}_{1} e^{-i \omega_{1} t}+\hat{\sigma}_{1}^{\dagger} e^{i \omega_{1} t}\right) \hat{E}\left[t, z_{1}(t)\right], \\
\hat{V}_{2}(t)=f(t) g\left(\hat{\sigma}_{2} e^{-i \omega_{2} t}+\hat{\sigma}_{2}^{\dagger} e^{i \omega_{2} t}\right) \hat{E}\left[t, z_{2}(t)\right],
\end{gathered}
$$

$\hat{E}(t, \mathbf{r})$ is the analog of the electric-field operator,

$$
\hat{E}(t, \mathbf{r})=\frac{\partial \hat{\Phi}(t, \mathbf{r})}{\partial t},
$$

and $\Phi(t, \mathbf{r})$ is a scalar field. In these equations $\hat{\sigma}$ and $\hat{\sigma}^{\dagger}$ are the atomic lowering and raising operators, and $z_{1}(t)$ and $z_{2}(t)$ 
are trajectories of atoms 1 and 2. If we choose plane waves as mode functions then

$$
\hat{\Phi}(t, \mathbf{r})=\sum_{\mathbf{k}} \frac{1}{\sqrt{2 v_{k}}}\left(\hat{a}_{\mathbf{k}} e^{-i v_{k} t+i \mathbf{k} \cdot \mathbf{r}}+\hat{a}_{\mathbf{k}}^{\dagger} e^{i v_{k} t-i \mathbf{k} \cdot \mathbf{r}}\right),
$$

where $v_{k}=c k$.

The evolution operator of the system obeys equation,

$$
i \hbar \frac{\partial \hat{U}(t)}{\partial t}=\hat{V}(t) \hat{U}(t)
$$

with the initial condition $\hat{U}\left(t_{0}\right)=1$. tions,

We introduce operators $\hat{U}_{1}(t)$ and $\hat{U}_{2}(t)$ which satisfy equa-

$$
\begin{aligned}
& i \hbar \frac{\partial \hat{U}_{1}(t)}{\partial t}=\hat{V}_{1}(t) \hat{U}_{1}(t), \\
& i \hbar \frac{\partial \hat{U}_{2}(t)}{\partial t}=\hat{V}_{2}(t) \hat{U}_{2}(t),
\end{aligned}
$$

and the same initial conditions $\hat{U}_{1,2}\left(t_{0}\right)=1$. The physical meaning of $\hat{U}_{1}(t)$ and $\hat{U}_{2}(t)$ is the following. $\hat{U}_{1}(t)$ is the evolution operator of the system if the interaction between field and atom 2 is turned off, that is, only atom 1 interacts with the field. $\hat{U}_{2}(t)$ is the evolution operator if the interaction between field and atom 1 is turned off, that is only atom 2 interacts with the field.

Next we show that in the second order in the interaction $\hat{V}$, the evolution operator of system $\hat{U}(t)$ can be written as

$$
\hat{U}(t) \approx \hat{U}_{1}(t) \hat{U}_{2}(t)+\frac{1}{\hbar^{2}} \int_{t_{0}}^{t} d t^{\prime \prime} \int_{t_{0}}^{t^{\prime \prime}} d t^{\prime}\left[\hat{V}_{1}\left(t^{\prime}\right), \hat{V}_{2}\left(t^{\prime \prime}\right)\right]
$$

Indeed, let us consider the difference,

$$
\hat{\Delta}(t)=\hat{U}(t)-\hat{U}_{1}(t) \hat{U}_{2}(t) .
$$

Using Eqs. (23)-(25), we obtain

$$
\begin{aligned}
\hat{\Delta}(t)= & \int_{t_{0}}^{t} d t^{\prime \prime} \frac{\partial \hat{\Delta}\left(t^{\prime \prime}\right)}{\partial t^{\prime \prime}} \\
= & -\frac{i}{\hbar} \int_{t_{0}}^{t} d t^{\prime \prime}\left\{\hat{V}\left(t^{\prime \prime}\right) \hat{U}\left(t^{\prime \prime}\right)\right. \\
& \left.-\hat{V}_{1}\left(t^{\prime \prime}\right) \hat{U}_{1}\left(t^{\prime \prime}\right) \hat{U}_{2}\left(t^{\prime \prime}\right)-\hat{U}_{1}\left(t^{\prime \prime}\right) \hat{V}_{2}\left(t^{\prime \prime}\right) \hat{U}_{2}\left(t^{\prime \prime}\right)\right\} .
\end{aligned}
$$

With the required accuracy one can replace $\hat{U}\left(t^{\prime \prime}\right) \approx$ $\hat{U}_{1}\left(t^{\prime \prime}\right) \hat{U}_{2}\left(t^{\prime \prime}\right)$ under the integral. Then we have

$$
\hat{\Delta}(t) \approx \frac{i}{\hbar} \int_{t_{0}}^{t} d t^{\prime \prime}\left[\hat{U}_{1}\left(t^{\prime \prime}\right), \hat{V}_{2}\left(t^{\prime \prime}\right)\right] \hat{U}_{2}\left(t^{\prime \prime}\right) .
$$

In the second order one can take under the integral,

$$
\hat{U}_{1}\left(t^{\prime \prime}\right) \approx 1-\frac{i}{\hbar} \int_{t_{0}}^{t^{\prime \prime}} d t^{\prime} \hat{V}_{1}\left(t^{\prime}\right)
$$

and $\hat{U}_{2}\left(t^{\prime \prime}\right) \approx 1$, which gives

$$
\hat{\Delta}(t) \approx \frac{1}{\hbar^{2}} \int_{t_{0}}^{t} d t^{\prime \prime} \int_{t_{0}}^{t^{\prime \prime}} d t^{\prime}\left[\hat{V}_{1}\left(t^{\prime}\right), \hat{V}_{2}\left(t^{\prime \prime}\right)\right] .
$$

Thus, Eq. (26) is correct in the second order.

Equation (26) is useful to study the problem of causality which we discuss next.

\section{PHOTON EMISSION AND CAUSALITY}

Let us assume that atom 1 emits a photon, e.g., by the acceleration radiation mechanism. Here we show that the excitation probability of detector atom 2 is independent of the presence of atom 1 if at the moment of detection the space-time position of the detector $\left(t, z_{2}\right)$ is not causally connected with the trajectory of atom 1 . To be specific, we assume that initially (at $t_{0}=-\infty$ ) both atoms are in ground-state $b$ and the field is in Minkowski vacuum state $\left|0_{M}\right\rangle$. That is, the initial-state vector of the system is

$$
\left|\psi_{0}\right\rangle=\left|b_{1} b_{2} 0\right\rangle .
$$

The evolution of the two-atom system is governed by the evolution operator (26). The state vector of the system at time $t$ can be written as

$$
|\psi(t)\rangle=\left|\psi_{b_{2}}(t)\right\rangle\left|b_{2}\right\rangle+\left|\psi_{a_{2}}(t)\right\rangle\left|a_{2}\right\rangle,
$$

where $\left|\psi_{b_{2}}(t)\right\rangle$ and $\left|\psi_{a_{2}}(t)\right\rangle$ are state vectors of atom 1 and the field provided that atom 2 is in states $b_{2}$ and $a_{2}$, respectively. The two states on the right-hand side of Eq. (29) are orthogonal. The probability that at time $t$ detector atom 2 is in excited-state $a_{2}$ is given by

$$
P_{2}(t)=\left\langle\psi_{a_{2}}(t) \mid \psi_{a_{2}}(t)\right\rangle .
$$

Using Eq. (26) and

$$
\hat{U}_{2}(t)=1-\frac{i}{\hbar} \int_{-\infty}^{t} d t^{\prime} \hat{V}_{2}\left(t^{\prime}\right)+\cdots,
$$

in the second order in the interaction we find

$$
\begin{aligned}
\left|\psi_{a_{2}}(t)\right\rangle\left|a_{2}\right\rangle= & \left(-\frac{i}{\hbar} \hat{U}_{1}(t) \int_{-\infty}^{t} d t^{\prime} \hat{V}_{2}\left(t^{\prime}\right)\right. \\
& \left.+\frac{1}{\hbar^{2}} \int_{t_{0}}^{t} d t^{\prime \prime} \int_{t_{0}}^{t^{\prime \prime}} d t^{\prime}\left[\hat{V}_{1}\left(t^{\prime}\right), \hat{V}_{2}\left(t^{\prime \prime}\right)\right]\right)\left|b_{1} b_{2} 0\right\rangle .
\end{aligned}
$$

If atoms 1 and 2 are not causally connected, then the commutator term in Eq. (31) does not contribute. Indeed, for the one-dimensional problem, the commutator of two electricfield operators is given by the derivative of the $\delta$ functions corresponding to the right and left propagating modes (see Appendix C),

$$
\begin{aligned}
{\left[\hat{E}\left(t^{\prime}, z_{1}\right), \hat{E}\left(t^{\prime \prime}, z_{2}\right)\right]=} & \frac{i c}{2} \frac{\partial}{\partial z_{2}}\left(\delta\left[c\left(t^{\prime}-t^{\prime \prime}\right)+z_{2}-z_{1}\right]\right. \\
& \left.-\delta\left[c\left(t^{\prime}-t^{\prime \prime}\right)+z_{1}-z_{2}\right]\right) .
\end{aligned}
$$

The term involving $\delta\left[c\left(t^{\prime}-t^{\prime \prime}\right)+z_{1}-z_{2}\right]$ shows that the probability of photon absorption by the second atom at the space-time point $\left(t^{\prime \prime}, z_{2}\right)$ is nonzero only if there is time $t^{\prime}$ such that along the first atom trajectory the equation,

$$
t^{\prime \prime}-t^{\prime}=\frac{1}{c}\left(z_{1}-z_{2}\right)
$$

has a solution. Physically this means that photon absorbed by the second atom at $\left(t^{\prime \prime}, z_{2}\right)$ was emitted by the first atom at a space-time point $\left(t^{\prime}, z_{1}\right)$ along the first atom trajectory and was propagating to the left with the speed of light until the moment of absorption (see Fig. 3). 


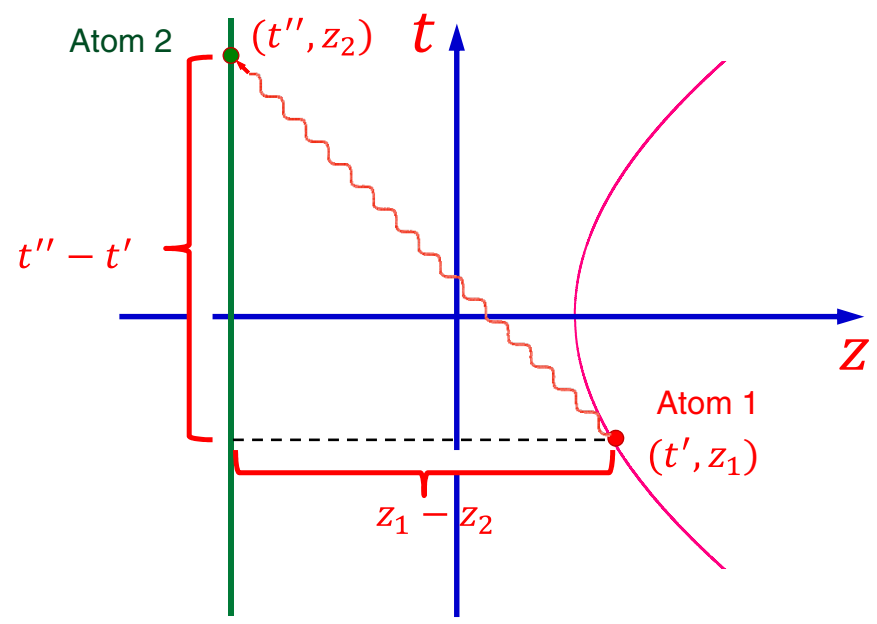

FIG. 3. Photon absorbed by atom 2 at a space-time point $\left(t^{\prime \prime}, z_{2}\right)$ was emitted by atom 1 at a space-time point $\left(t^{\prime}, z_{1}\right)$ along atom's 1 trajectory and was propagating with the speed of light $c$ until the moment of absorption. The photon's trajectory is shown as a wavy line with the slope $1 / c$.

Thus, if atoms 1 and 2 are not causally connected, we obtain

$$
\left|\psi_{a_{2}}(t)\right|\left|a_{2}\right\rangle \approx-\frac{i}{\hbar} \hat{U}_{1}(t) \int_{-\infty}^{t} d t^{\prime} \hat{V}_{2}\left(t^{\prime}\right)\left|b_{1} b_{2} 0\right\rangle .
$$

Therefore, the probability of the detector atom excitation is given by

$$
\begin{aligned}
P_{a_{2}}(t)= & \left\langle\psi_{a_{2}}(t) \mid \psi_{a_{2}}(t)\right\rangle \\
= & \frac{1}{\hbar^{2}}\left\langle b_{1} b_{2} 0\right| \int_{-\infty}^{t} d t^{\prime} \hat{V}_{2}\left(t^{\prime}\right) \hat{U}_{1}^{\dagger}(t) \hat{U}_{1}(t) \\
& \times \int_{-\infty}^{t} d t^{\prime \prime} \hat{V}_{2}\left(t^{\prime \prime}\right)\left|b_{1} b_{2} 0\right\rangle .
\end{aligned}
$$

Using unitarity,

$$
\hat{U}_{1}^{\dagger}(t) \hat{U}_{1}(t)=1
$$

we obtain

$$
P_{a_{2}}(t)=\frac{1}{\hbar^{2}}\left\langle b_{2} 0\left|\int_{-\infty}^{t} d t^{\prime} \hat{V}_{2}\left(t^{\prime}\right) \int_{-\infty}^{t} d t^{\prime \prime} \hat{V}_{2}\left(t^{\prime \prime}\right)\right| b_{2} 0\right\rangle .
$$

The matrix element does not involve operator $\hat{V}_{1}$. Therefore, if detector atom 2 performs a measurement at a space-time point which is causally disconnected from atom 1 , then the excitation probability of atom 2 is independent of the presence of atom 1 . This is true, in general, when the evolution operator of the system can be written as $\hat{U}(t)=\hat{U}_{1}(t) \hat{U}_{2}(t)$.

In particular, this result yields that the right-propagating photon emitted by atom 1 cannot excite detector atom 2 located to the left from atom 1 even though the mode function of the emitted photon extends into the left region [22].

If detector atom 2 is stationary it is causally connected with emitter atom 1 in the future Rindler wedge $c t>|z|$ by the left-propagating photons which can excite the detector (see Fig. 1). However, if the interaction between the detector atom and the field is turned off before the atom's trajectory enters the future Rindler wedge (see Fig. 4) detector atom 2 is no

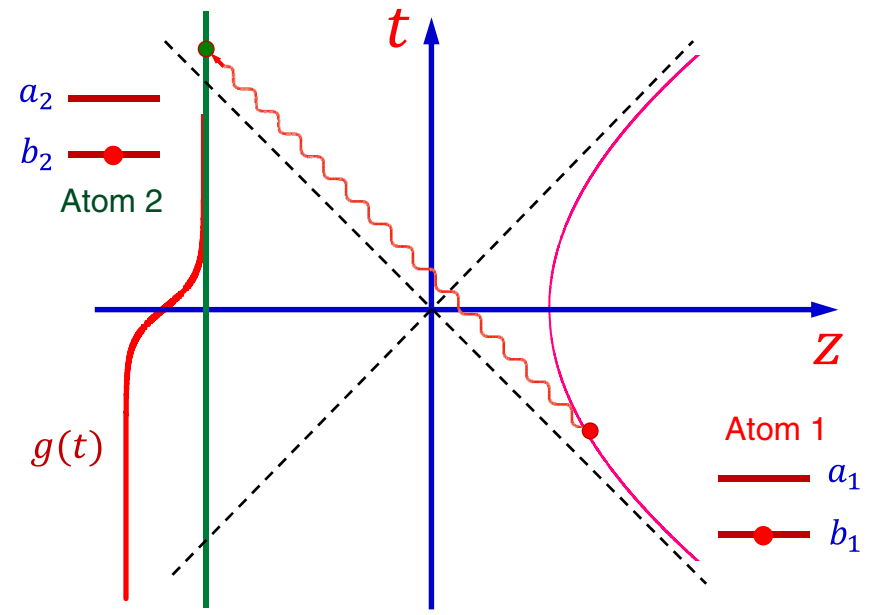

FIG. 4. Coupling between detector atom 2 and field $g(t)$ is adiabatically switched off before the atom enters the future Rindler wedge $c t>|z|$.

longer causally connected with photons emitted by atom 1 . If the switching is adiabatic, then the change in the coupling constant with time does not yield excitation of the detector atom, and the atom will remain in the ground state.

\section{PROBABILITY OF JOINT ATOM EXCITATION AND VACUUM ENTANGLEMENT}

In the previous section we showed that probability of detector atom excitation $P_{a_{2}}$ is independent of emitter atom 1 if the atoms are located in the causally disconnected regions. The detector atom can get self-excited, e.g., by nonadiabatic switching of the coupling between the atom and the field, by moving with acceleration, or by other mechanisms.

One can write $P_{a_{2}}$ as

$$
P_{a_{2}}=P_{a_{2} b_{1}}+P_{a_{2} a_{1}},
$$

where $P_{a_{2} b_{1}}$ and $P_{a_{2} a_{1}}$ are conditional probabilities that detector atom 2 becomes excited and atom 1 is in the ground $\left(b_{1}\right)$ and excited $\left(a_{1}\right)$ states, respectively. Due to quantum correlations (vacuum entanglement) even if the two atoms are not causally connected, the conditional probability $P_{a_{2} a_{1}}$ can be nonseparable, that is, $P_{a_{2} a_{1}} \neq P_{a_{2}} P_{a_{1}}$. This, however, does not contradict causality.

To clarify this issue we consider atoms 1 and 2 which are uniformly accelerated in the opposite Rindler wedges with acceleration $a$ in Minkowski vacuum (see Fig. 5). The atom's trajectories are causally disconnected, that is, the signal emitted by one of the atoms and propagating with the speed of light, cannot reach the other atom. We assume that initially both atoms are in the ground state and the field is in Minkowski vacuum.

A ground-state atom having transition frequency $\omega$ moving in the right Rindler wedge with acceleration $a$ emits a left-propagating photon into the Unruh-Minkowski mode $F_{1 \omega}$ and a right-propagating photon into the mode $F_{2 \omega}$ (see Appendix B). An atom accelerated in the left Rindler wedge emits a right-propagating photon into the mode $F_{1 \omega}$ and a left-propagating photon into the mode $F_{2 \omega}$. That is, atoms 1 


\section{Minkowski space}

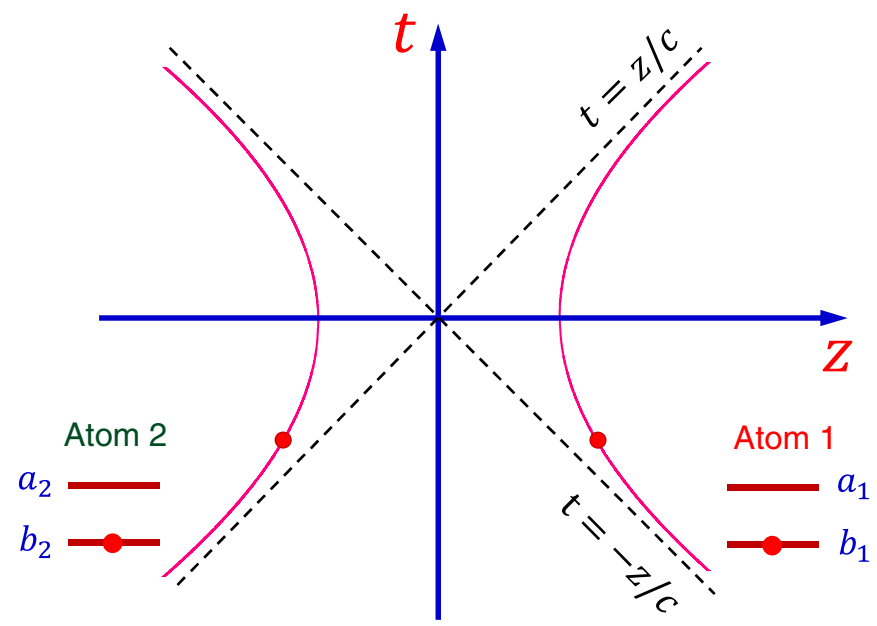

FIG. 5. Ground-state atoms 1 and 2 are uniformly accelerated in the right and left Rindler wedges, respectively, and become excited simultaneously with probability $P_{a_{1} a_{2}}$.

and 2 emit photons into different Unruh-Minkowski modes and, hence, the probability amplitudes of these processes do not interfere. As a result, the conditional probability that both atoms become excited and the two Unruh-Minkowski photons are emitted is equal to $P_{a_{2}} P_{a_{1}}$.

However, joint excitation of the atoms can also leave the state of the field in Minkowski vacuum $\left|0_{M}\right\rangle$. We denote the corresponding conditional probability as $P_{a_{2} a_{1} 0}$ and obtain

$$
P_{a_{2} a_{1}}=P_{a_{2}} P_{a_{1}}+P_{a_{2} a_{1} 0} .
$$

In Appendix D we show that $P_{a_{2} a_{1} 0}$ vanishes if the accelerated atoms have different transition frequencies $\omega_{1} \neq \omega_{2}$, however, for $\omega_{1}=\omega_{2}=\omega$,

$$
P_{a_{2} a_{1} 0}=e^{2 \pi c \omega / a} P_{a_{2}} P_{a_{1}} .
$$

That is, $P_{a_{2} a_{1} 0}>P_{a_{2}} P_{a_{1}}$ for $\omega_{1}=\omega_{2}$ and, hence, $P_{a_{2} a_{1}}>$ $P_{a_{2}} P_{a_{1}}$. The fact that $P_{a_{2} a_{1}}$ can be greater than $P_{a_{2}} P_{a_{1}}$ if the atoms are not causally connected is a manifestation of vacuum correlations (entanglement).

If both atoms become excited, the final state of the field $\left|\psi_{a_{2} a_{1}}\right\rangle$ overlaps with the initial field state (Minkowski vacuum) provided $\omega_{1}=\omega_{2}$. According to Appendix D,

$$
\left|\psi_{a_{2} a_{1}}\right\rangle \propto\left(\hat{b}_{R 1 \omega_{1}}+\hat{b}_{L 2 \omega_{1}}\right)\left(\hat{b}_{R 2 \omega_{2}}+\hat{b}_{L 1 \omega_{2}}\right)\left|0_{M}\right\rangle,
$$

where $\hat{b}_{v}$ 's are annihilation operators of the Rindler photons which are described by the mode functions (5) and (6). The indices $R$ and $L$ refer to the right- and the left-propagating photons, respectively. Recall that Minkowski vacuum is filled with Rindler photons and, according to Eq. (36), the atoms become excited by absorbing Rindler photons.

The operator $\hat{b}_{R 2 \omega_{2}}+\hat{b}_{L 1 \omega_{2}}$ describes annihilation of the right- and left-propagating Rindler photons which are causally connected with atom 2 , whereas $\hat{b}_{R 1 \omega_{1}}+\hat{b}_{L 2 \omega_{1}}$ describes annihilation of the Rindler photons which are causally connected with atom 1. Each atom becomes excited by absorbing Rindler photons with the mode functions which are restricted to the region causally connected with the atom. This agrees with causality.

However, the process of absorption of a pair of Rindler photons, described by the operators $\hat{b}_{R 1 \omega} \hat{b}_{R 2 \omega}$ or $\hat{b}_{L 2 \omega} \hat{b}_{L 1 \omega}$, yields a state of the field which overlaps with the initial-state $\left|0_{M}\right\rangle$. As a consequence, the conditional probability $P_{a_{2} a_{1} 0}$ is nonzero.

In the Minkowski vacuum state the number of Rindler photons in the modes $\phi_{1 v}$ and $\phi_{2 v}$ is correlated. Using relations (A7) and (A8) we obtain the following representation of Minkowski vacuum in terms of Rindler states (see Appendix E),

$$
\begin{aligned}
\left|0_{M}\right\rangle & =\prod_{\nu>0}\left(1-e^{-2 \pi c v / a}\right) \exp \left[\exp \left(-\frac{\pi c v}{a}\right)\left(\hat{b}_{R 1 v}^{\dagger} \hat{b}_{R 2 v}^{\dagger}+\hat{b}_{L 1 v}^{\dagger} \hat{b}_{L 2 v}^{\dagger}\right)\right]\left|0_{R}\right\rangle \\
& =\prod_{\nu>0}\left(1-e^{-2 \pi c v / a}\right) \sum_{\substack{n_{R 1 v}=n_{R 2 v}=0, n_{L 1 v}=n_{L 2 v}=0}}^{\infty} e^{-\pi\left(n_{R 1 v}+n_{L 1 v}\right) c v / a}\left|n_{R 1 v}, n_{R 2 v}\right\rangle\left|n_{L 1 v}, n_{L 2 v}\right\rangle,
\end{aligned}
$$

where $\left|n_{R 1 v}, n_{R 2 v}\right\rangle\left(\left|n_{L 1 v}, n_{L 2 v}\right\rangle\right)$ are states with $n_{R 1 v}$ and $n_{R 2 v}$ $\left(n_{L 1 v}\right.$ and $\left.n_{L 2 v}\right)$ Rindler photons in the right- (left-) propagating modes $\phi_{1 v}$ and $\phi_{2 v}$.

Due to vacuum correlations, if detector atom 2 becomes excited by absorbing the right-propagating Rindler photon $\phi_{2 \omega}$ of frequency $\omega$ then with unit probability there is the nonzero number of the right-propagating Rindler photons in mode $\phi_{1 \omega}$. They excite atom 1 . That is, atom 1 becomes excited with a much higher probability provided that atom 2 detected a photon. This is the reason why $P_{a_{2} a_{1}}>P_{a_{2}} P_{a_{1}}$.

The same physical argument yields $P_{a_{2} b_{1}}<P_{a_{2}} P_{b_{1}}$. Namely, if atom 2 detected a photon then with unit probability there is a nonzero number of Rindler photons which can excite atom 1 and, thus, it is less likely that atom 1 will remain in the ground state. However, the sum $P_{a_{2} b_{1}}+P_{a_{2} a_{1}}$ is independent of atom 1 as it was shown in the previous section.

Using Eq. (14) the state of the field obtained by absorption of Rindler photons by two causally disconnected atoms in Minkowski vacuum can be written as

$$
\hat{b}_{1 \omega} \hat{b}_{2 \omega}\left|0_{M}\right\rangle=\frac{\hat{a}_{1 \omega} \hat{a}_{1 \omega}^{\dagger}+e^{-\pi c \omega / a} \hat{a}_{2 \omega}^{\dagger} \hat{a}_{1 \omega}^{\dagger}}{2 \sinh (\pi c \omega / a)}\left|0_{M}\right\rangle .
$$

The term $\hat{a}_{1 \omega} \hat{a}_{1 \omega}^{\dagger}\left|0_{M}\right\rangle$ on the right-hand side of Eq. (38) can be interpreted as emission of the Unruh-Minkowski photon $F_{1 \omega}$ by one atom followed by absorption of this photon by the other atom. Such interpretation implies that causally 


\section{Minkowski space}

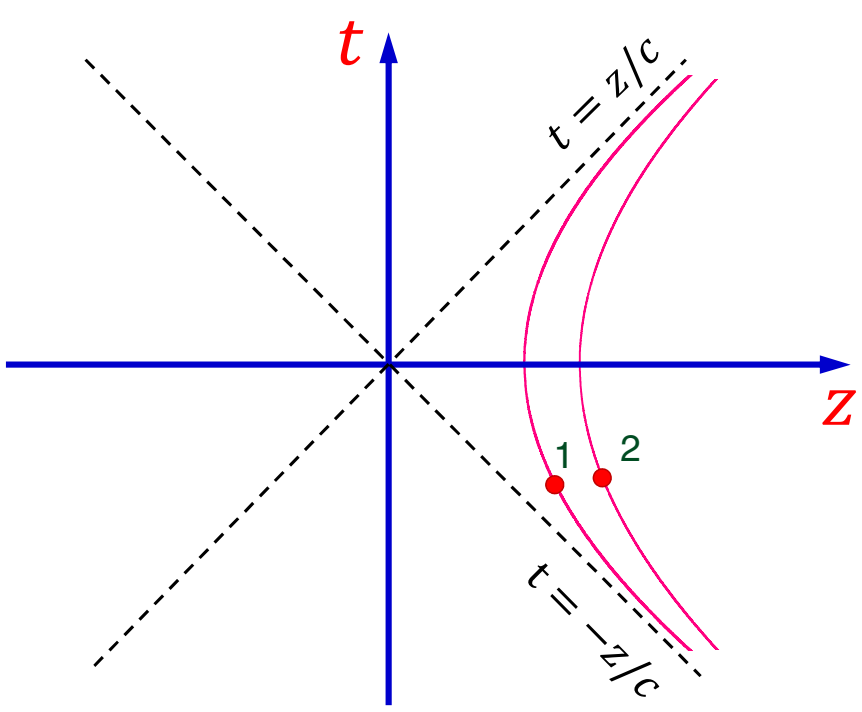

FIG. 6. Ground-state atoms 1 and 2 are uniformly accelerated in the right Rindler wedge in Minkowski vacuum and become excited.

disconnected atoms can excite each other which is at odds with causality. However, the term $\hat{a}_{1 \omega} \hat{a}_{1 \omega}^{\dagger}\left|0_{M}\right\rangle$ cannot be considered separately from the other term $\hat{a}_{2 \omega}^{\dagger} \hat{a}_{1 \omega}^{\dagger}\left|0_{M}\right\rangle$ describing emission of two Unruh-Minkowski photons in modes $F_{1 \omega}$ and $F_{2 \omega}$. According to Eq. (38), the combination of these terms yields $\hat{b}_{1 \omega} \hat{b}_{2 \omega}\left|0_{M}\right\rangle$ which describes a causal process.

Finally we mention the case when identical atoms 1 and 2 are uniformly accelerated in the right Rindler wedge in Minkowski vacuum (see Fig. 6). In this case the atom's trajectories are causally connected. Atom 1 or 2 can become excited by emitting a photon into the Unruh-Minkowski mode. Can the other atom become excited by absorbing such a UnruhMinkowski photon? If such a process can occur, then the final state of the field would be Minkowski vacuum, and the conditional probability $P_{a_{2} a_{1} 0}$ would be nonzero.

It is easy to find $P_{a_{2} a_{1} 0}$ using a representation of Minkowski vacuum in terms of the Rindler states given by Eq. (38). In the Rindler picture Minkowski vacuum is filled with Rindler photons, and the accelerated atom becomes excited by absorbing such photons. If both atoms move in the right Rindler wedge, they can absorb photons only from the right-propagating modes $\phi_{R 1 v}$ or the left-propagating modes $\phi_{L 2 v}$. Each such process changes the Fock states $\left|n_{R v}, n_{R \nu}\right\rangle$ or $\left|n_{L v}, n_{L v}\right\rangle$ in Eq. (38) into $\left|n_{R v}-1, n_{R v}\right\rangle$ or $\left|n_{L v}, n_{L v}-1\right\rangle$, whereas the number of the Rindler photons in modes $\phi_{L 1 v}$ and $\phi_{R 2 v}$ does not change. As a consequence, if both atoms become excited, the state of the field does not contain Fock states of the form $\left|n_{R v}, n_{R v}\right\rangle\left|n_{L v}, n_{L v}\right\rangle$, and, hence, it is orthogonal to $\left|0_{M}\right\rangle$. That is if both atoms are uniformly accelerated in the same Rindler wedge, then the probability that both atoms become excited and the field remains in Minkowski vacuum is equal to zero $\left(P_{a_{2} a_{1} 0}=0\right)$.

This property can be understood from a negative frequency perspective. Namely, the Unruh-Minkowski photon emitted by a ground-state atom has negative frequency (negative energy) from the perspective of an atom accelerated in the same direction and, as a consequence, such a photon cannot be absorbed by the accelerated ground-state atom [23]. This does not mean that the presence of the other atom makes no difference. Each of the atoms as it is excited by the vacuum fluctuations emits an Unruh-Minkowski photon, and these emissions are largely independent of each other. There is, however, a probability, if both atoms have the same (redshifted) frequency, namely, $a_{1} \omega_{2}=a_{2} \omega_{1}$ that both will emit the photon in the same mode. In this case one will get a Dicke superradiance condition, and the probability that both emit into that same mode is larger by a factor of 2 than the square of the probability that both would emit into that mode independently.

Using Eq. (14) the state of the field obtained by absorption of identical Rindler photons by atoms moving in the same Rindler wedge with equal acceleration $a$ can be written as

$$
\hat{b}_{1 \omega} \hat{b}_{1 \omega}\left|0_{M}\right\rangle=\frac{e^{-\pi c \omega / a} \hat{a}_{2 \omega}^{\dagger} \hat{a}_{2 \omega}^{\dagger}}{2 \sinh (\pi c \omega / a)}\left|0_{M}\right\rangle .
$$

That is, the absorption process can be interpreted as the stimulated emission of two Unruh-Minkowski photons in the same mode. The emitted photons interfere constructively which yields a factor of $\sqrt{2}$ in the corresponding probability amplitude $\hat{a}_{2 \omega}^{\dagger} \hat{a}_{2 \omega}^{\dagger}\left|0_{M}\right\rangle=\sqrt{2}\left|2_{2 \omega}\right\rangle$. As a result, the probability of the same-wedge atom's simultaneous excitation without specifying the state of the field is

$$
P_{a_{2} a_{1}}=2 P_{a_{2}} P_{a_{1}} \text {. }
$$

This should be compared with the result we obtained for the atoms accelerated in the opposite wedges,

$$
P_{a_{2} a_{1}}=\left(e^{2 \pi c \omega / a}+1\right) P_{a_{2}} P_{a_{1}},
$$

which yields a larger value for the conditional probability.

\section{TESTING VACUUM ENTANGLEMENT WITH A $\delta$ FUNCTION DETECTOR}

Detector atom 2 can become self-excited if coupling between atom 2 and the field changes with time nonadiabatically. In this section we assume that atom 2 is fixed at the coordinate $z_{2}$ and its coupling with the field changes with time as a $\delta$ function. Namely, in the interaction Hamiltonian (20) we take $f(t)=\delta(t-T)$. Thus, the detector atom tests the state of the field at the space-time point $\left(T, z_{2}\right)$.

We assume that atom 1 with transition frequency $\omega_{1}$ is uniformly accelerated in Minkowski vacuum and moves along the trajectory (10) such that atom 1 is always located to the right of detector atom 2 (see Fig. 7). We assume that the field contains only right-propagating modes and coupling between atom 1 and the field is switched off adiabatically before $t=T$. Under these assumptions the two atoms are causally disconnected and the probability of their joint excitation can serve as a measure of entanglement of Minkowski vacuum.

Atom 1 can become excited by absorbing a rightpropagating Rindler photon in mode $\phi_{1 \omega_{1}}$. According to Eq. (38), absorption of such a photon by atom 1 implies that with unit probability there is a nonzero number of Rindler photons in the mode $\phi_{2 \omega_{1}}$ which can excite the detector 


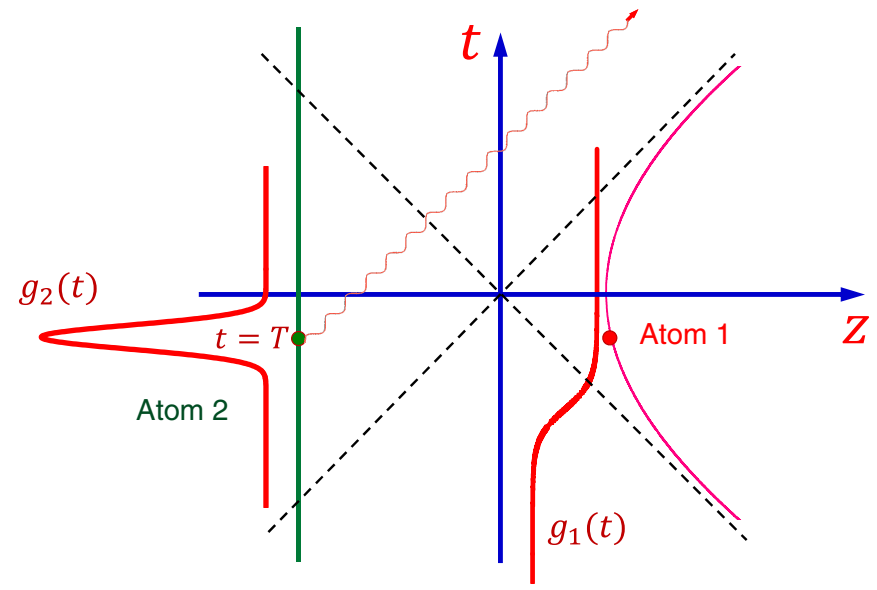

FIG. 7. Atom 1 is uniformly accelerated in the right Rindler wedge, whereas detector atom 2 is fixed to the left from atom 1 . The coupling between detector atom 2 and the field changes with time as $\delta(t-T)$, whereas the coupling between atom 1 and the field is switched off adiabatically before $t=T$. The field contains only right-propagating modes and initially the field is in Minkowski vacuum. The probability of atom's joint excitation serves as a measure of entanglement of Minkowski vacuum.

atom in the space-time region $t>z / c$. Thus, the conditional probability that both atoms become excited at time $T$ yields information about photon correlation in the Rindler-modes $\phi_{1 v}$ and $\phi_{2 v}$ in Minkowski vacuum. The right-propagating modes $\phi_{1 v}$ and $\phi_{2 v}$ are localized in the opposite sides of the line $t=z / c$ separating two causally disconnected regions. The $\delta$-function detector allows us to test the space-time dependence of such correlations.

In Appendix F we calculate the conditional probability that both atoms 1 and 2 are excited at time $T$ and the field is in Minkowski vacuum. We find that

$$
P_{a_{2} a_{1} 0}\left(T, z_{2}\right) \propto \frac{e^{-\left[\left(2 \pi c \omega_{1}\right) / a\right] \theta\left(z_{2} / c-T\right)}}{\left(z_{2} / c-T\right)^{4}} .
$$

Equation (42) shows that conditional probability $P_{a_{2} a_{1} 0}\left(T, z_{2}\right)$ is in a factor $\exp \left(2 \pi c \omega_{1} / a\right)$ larger if detector atom 2 is located in the region $T>z_{2} / c$ which is causally disconnected from accelerated atom 1 . The degree of correlations depends on the proximity of the detector atom to the Rindler horizon $T=z_{2} / c$. Namely, the conditional probability $P_{a_{2} a_{1} 0}$ formally diverges at $T=z_{2} / c$ and decays as a power law away from the horizon line.

\section{SUMMARY}

In this paper we show that the evolution of atoms located in causally disconnected space-time regions is independent of each other, that is, conventional quantum optical analysis yields causal dynamics. This is true, in general, when the evolution operator of the system can be factorized as $\hat{U}(t)=\hat{U}_{1}(t) \hat{U}_{2}(t)$. The result can be applied, e.g., to Unruh acceleration radiation [14] or to the Fermi problem [11]. The latter deals with the issue of causality in spontaneous emission of a photon by a stationary atom.
The description of a state of the quantized electromagnetic field in terms of photons depends on a choice of the basis set for the field mode functions. As a consequence, the particle content of Minkowski vacuum $\left|0_{M}\right\rangle$ depends on the mode functions we adopt to describe photons. For a plane-wave basis set of the form

$$
f_{v}(t, z)=\frac{1}{\sqrt{2 v}} e^{-i v(t \pm z / c)}, \quad v>0,
$$

the state of Minkowski vacuum is a state with no photons in the modes (43). The mode functions (43) are nonzero in the entire Minkowski space-time.

However, if, e.g., we choose Rindler-modes (5) and (6) as a basis set, then in such a description, Minkowski vacuum is filled with Rindler photons [see Eq. (38)]. The Rindlermodes (5) and (6) are nonzero in the half-plane of Minkowski space-time. According to Eq. (38), the number of photons in the Rindler-modes $\phi_{1 v}$ and $\phi_{2 v}$ is correlated in Minkowski vacuum. Namely, the photon numbers in the right- (left-) propagating modes $\phi_{1 v}$ and $\phi_{2 v}$ are equal. This correlation (entanglement) property of Minkowski vacuum described in terms of the Rindler photons yields observable effects. Since a uniformly accelerated atom can be excited by absorbing a Rindler photon, such atoms can be used as a tool to test Rindler photon content and correlations of Minkowski vacuum.

For example, ground-state atoms 1 and 2 uniformly accelerated in Minkowski vacuum in the opposite Rindler wedges (see Fig. 5) are causally disconnected. However, they can become excited simultaneously with a probability $P_{a_{2} a_{1}}$ much greater than $P_{a_{2}} P_{a_{1}}$, where $P_{a_{2}}$ and $P_{a_{1}}$ are the excitation probabilities of atoms 2 and 1 independently. This result does not violate causality and can be understood as follows. In the Rindler-mode picture, atom 1 becomes excited by absorbing the Rindler photon from the right Rindler wedge. Because of Minkowski vacuum correlations this implies that with unit probability there is nonzero number of photons in the left Rindler wedge and these photons excite atom 2. As a result, the probability of joint excitation $P_{a_{2} a_{1}}$ can be greater than $P_{a_{2}} P_{a_{1}}$.

Interpretation of this result in the Unruh-Minkowski picture might lead to an impression that causality is violated. This is the case because Unruh-Minkowski modes (12) and (13) extend in the region causally disconnected with the atom. In the Unruh-Minkowski picture, an accelerated atom becomes excited by emitting the Unruh-Minkowski photon which then can be absorbed by the causally disconnected atom accelerated in the opposite Rindler wedge. Emission of a photon followed by its absorption in a causally disconnected region might be interpreted as acausal dynamics. However, in the Unruh-Minkowski picture there is another process which leads to the simultaneous excitation of both atoms. Namely, each atom can become excited independently by emitting the Unruh-Minkowski photon so that the final state of the field has two Unruh-Minkowski photons. According to Eq. (38), the sum of these processes is equivalent to absorption of Rindler photons from the causally connected regions, that is, summing these two terms leads to causal dynamics.

Another interesting feature of Minkowski vacuum entanglement is that atoms uniformly accelerated in the same 
Rindler wedge (see Fig. 6) cannot become simultaneously excited without changing the state of the field. That is that the Unruh-Minkowski photon emitted by one atom cannot be absorbed by another atom accelerated in the same direction [23]. One might expect that one of the atoms would become excited by absorbing the Unruh-Minkowski photon that the other atom emitted. But it does not, both atoms emit photons, leaving the state of the field with two particles rather than none, if it were absorbed.

In principle, entanglement of Minkowski vacuum can be harvested [24]. For example, entanglement can be extracted from the vacuum, delivered to the atoms, and distilled into Einstein, Podolsky, and Rosen pairs used in quantum information tasks [25]. Therefore, teleportation and other entanglement assisted quantum communication tasks can rely on the vacuum alone as a resource for entanglement [25,26]. Recently entanglement harvesting protocols have been applied to spacelike [27] and timelike [28,29] separated detectors as well as situations involving uniform accelerations [30].

\section{ACKNOWLEDGMENTS}

This work was supported by the Air Force Office of Scientific Research (Grant No. FA9550-20-1-0366 DEF), the Office of Naval Research (Grants No. N00014-20-1-2184 and No. N00014-16-1-2578), the Robert A. Welch Foundation (Grant No. A-1261), the National Science Foundation (Grant No. PHY-2013771), and the Natural Sciences and Engineering Research Council of Canada (NSERC). W.U. thanks the TAMU Hagler Institute for Advanced Studies for their support.

\section{APPENDIX A: RELATION BETWEEN RINDLER AND UNRUH-MINKOWSKI MODE OPERATORS}

To be specific, we consider only the right-propagating modes. In terms of the Rindler-modes (5) and (6) the field operator for the right-propagating photons reads

$$
\hat{\Phi}=\int_{0}^{\infty} d v\left(\phi_{1 v} \hat{b}_{1 v}+\phi_{1 v}^{*} \hat{b}_{1 v}^{\dagger}+\phi_{2 v} \hat{b}_{2 v}+\phi_{2 v}^{*} \hat{b}_{2 v}^{\dagger}\right)
$$

The right-propagating Unruh-Minkowski modes are defined as

$$
\begin{aligned}
& F_{1 v}=\frac{\phi_{1 v}+e^{-\pi c v / a} \phi_{2 v}^{*}}{\sqrt{1-e^{-2 \pi c v / a}}}, \\
& F_{2 v}=\frac{\phi_{2 v}+e^{-\pi c v / a} \phi_{1 v}^{*}}{\sqrt{1-e^{-2 \pi c v / a}}} .
\end{aligned}
$$

Modes $F_{1 v}$ and $F_{2 v}$ are normalized in the same way as the Rindler modes and are orthogonal to each other $\left\langle F_{1 v} \mid F_{2 v}\right\rangle=0$. From Eqs. (A2) and (A3) we obtain

$$
\begin{aligned}
& \phi_{1 v}=\frac{F_{1 v}-e^{-\pi c v / a} F_{2 v}^{*}}{\sqrt{1-e^{-2 \pi c v / a}}}, \\
& \phi_{2 v}=\frac{F_{2 v}-e^{-\pi c v / a} F_{1 v}^{*}}{\sqrt{1-e^{-2 \pi c v / a}}} .
\end{aligned}
$$

Plugging this into Eq. (A1) and combining terms we find

$$
\hat{\Phi}=\int_{0}^{\infty} d v\left(F_{1 v} \frac{\hat{b}_{1 v}-e^{-\pi c v / a} \hat{b}_{2 v}^{\dagger}}{\sqrt{1-e^{-2 \pi c v / a}}}+F_{1 v}^{*} \frac{\hat{b}_{1 v}^{\dagger}-e^{-\pi c v / a} \hat{b}_{2 v}}{\sqrt{1-e^{-2 \pi c v / a}}}+F_{2 v} \frac{\hat{b}_{2 v}-e^{-\pi c v / a} \hat{b}_{1 v}^{\dagger}}{\sqrt{1-e^{-2 \pi c v / a}}}+F_{2 v}^{*} \frac{\hat{b}_{2 v}^{\dagger}-e^{-\pi c v / a} \hat{b}_{1 v}}{\sqrt{1-e^{-2 \pi c v / a}}}\right) .
$$

The operators in front of $F_{1 v}, F_{1 v}^{*}, F_{2 v}$, and $F_{2 v}^{*}$ are associated with the Unruh-Minkowski mode operators $\hat{a}_{1 v}, \hat{a}_{1 v}^{\dagger}$, $\hat{a}_{2 v}$, and $\hat{a}_{2 v}^{\dagger}$, respectively. Therefore,

$$
\begin{aligned}
& \hat{a}_{1 v}=\frac{\hat{b}_{1 v}-e^{-\pi c v / a} \hat{b}_{2 v}^{\dagger}}{\sqrt{1-e^{-2 \pi c v / a}}}, \quad \hat{a}_{1 v}^{\dagger}=\frac{\hat{b}_{1 v}^{\dagger}-e^{-\pi c v / a} \hat{b}_{2 v}}{\sqrt{1-e^{-2 \pi c v / a}}}, \\
& \hat{a}_{2 v}=\frac{\hat{b}_{2 v}-e^{-\pi c v / a} \hat{b}_{1 v}^{\dagger}}{\sqrt{1-e^{-2 \pi c v / a}}}, \quad \hat{a}_{2 v}^{\dagger}=\frac{\hat{b}_{2 v}^{\dagger}-e^{-\pi c v / a} \hat{b}_{1 v}}{\sqrt{1-e^{-2 \pi c v / a}}} .
\end{aligned}
$$

These expressions can be inverted which yields relations between operators for the Rindler-modes $\hat{b}_{v}$ and the Unruh-Minkowski modes $\hat{a}_{v}$,

$$
\hat{b}_{1 v}=\frac{\hat{a}_{1 v}+e^{-\pi c v / a} \hat{a}_{2 v}^{\dagger}}{\sqrt{1-e^{-2 \pi c v / a}}}, \quad \hat{b}_{2 v}=\frac{\hat{a}_{2 v}+e^{-\pi c v / a} \hat{a}_{1 v}^{\dagger}}{\sqrt{1-e^{-2 \pi c v / a}}}
$$

\section{APPENDIX B: PHOTON EMISSION BY A UNIFORMLY ACCELERATED ATOM}

Here we consider electrically neutral two-level ( $a$ and $b$ ) atom with transition angular frequency $\omega$ which is uniformly accelerated along the trajectory (10). Initially the field is in Minkowski vacuum, and the atom is in the ground state. We calculate 
the mode into which the atom emits a photon. We quantize the field using the right- and left-moving plane waves in Minkowski space-time as a basis set,

$$
f_{v R}(t, z)=\frac{1}{\sqrt{2 v}} e^{-i v(t-z / c)}, \quad f_{v L}(t, z)=\frac{1}{\sqrt{2 v}} e^{-i v(t+z / c)},
$$

and assume that the field is scalar. The field operator in terms of $f_{v R}(t, z)$ and $f_{v L}(t, z)$ reads

$$
\hat{\Phi}(t, z)=\int_{0}^{\infty} d v\left[\hat{a}_{v R} f_{v R}(t, z)+\hat{a}_{v R}^{\dagger} f_{v R}^{*}(t, z)+\hat{a}_{v L} f_{v L}(t, z)+\hat{a}_{v L}^{\dagger} f_{v L}^{*}(t, z)\right],
$$

where $\hat{a}_{v R}^{\dagger}$ and $\hat{a}_{v L}^{\dagger}$ are creation operators of the right- and left-propagating plane-wave photons with frequency $v$. We will assume the following form of the interaction Hamiltonian between the atom and the scalar field:

$$
\hat{V}(\tau)=g\left(\hat{\sigma} e^{-i \omega \tau}+\hat{\sigma}^{\dagger} e^{i \omega \tau}\right) \frac{\partial}{\partial \tau} \hat{\Phi}[t(\tau), z(\tau)],
$$

where $g$ is the atom-field coupling constant and $\hat{\sigma}$ is the atomic lowering operator. Since the atom feels the local value of the field the operator $\hat{\Phi}$ is taken at the atom's position $t(\tau), z(\tau)$. The probability amplitude that the atom becomes excited, and the photon is emitted into the right-moving mode $f_{v R}(t, z)$ is given by the matrix element,

$$
A_{\nu R}=-\frac{i}{\hbar}\left\langle a 1_{\nu R}\left|\int_{-\infty}^{\infty} d \tau \hat{V}(\tau)\right| b 0\right\rangle=-\frac{i g}{\hbar} \int_{-\infty}^{\infty} d \tau e^{i \omega \tau} \frac{\partial}{\partial \tau} f_{\nu R}^{*}[t(\tau), z(\tau)]=-\frac{i g}{\sqrt{2 \nu} \hbar} \int_{-\infty}^{\infty} d \tau e^{i \omega \tau} \frac{\partial}{\partial \tau} e^{i v[t(\tau)-z(\tau) / c]} .
$$

Taking into account that along the atom's trajectory,

$$
t(\tau)-z(\tau) / c=-\frac{c}{a} e^{-a \tau / c}
$$

we obtain

$$
A_{\nu R}=-\frac{i g}{\sqrt{2 v} \hbar} \int_{-\infty}^{\infty} d \tau e^{i \omega \tau} \frac{\partial}{\partial \tau} e^{-(i v c / a) e^{-a \tau / c}}=\frac{g \sqrt{v}}{\sqrt{2} \hbar} \int_{-\infty}^{\infty} d \tau e^{i \omega \tau} e^{-(i v c / a) e^{-a \tau / c}} e^{-a \tau / c} .
$$

To find the mode function $F_{R}(t, z)$ into which the photon is emitted we need to multiply $A_{v R}$ by $f_{v R}(t, z)$ and integrate over all mode frequencies $v$,

$$
F_{R}(t, z)=\int_{0}^{\infty} d v A_{v R} f_{v R}(t, z)=\frac{g}{2 \hbar} \int_{0}^{\infty} d v \int_{-\infty}^{\infty} d \tau e^{i \omega \tau} e^{-a \tau / c} e^{-(i v c / a) e^{-a \tau / c}} e^{-i v(t-z / c)} .
$$

The integral over frequency $v$ can be calculated using the formula,

$$
\int_{0}^{\infty} e^{i v t} d v=\frac{i}{t+i \lambda}
$$

where $\lambda \rightarrow 0+$. This yields

$$
F_{R}(t, z)=-\frac{i g}{2 \hbar} \int_{-\infty}^{\infty} d \tau \frac{e^{i \omega \tau} e^{-a \tau / c}}{u+\frac{c}{a} e^{-a \tau / c}-i \lambda},
$$

where

$$
u=t-z / c .
$$

The integration over proper time $\tau$ can be performed by closing the contour in the upper half of the complex plane and summing up contributions from the poles. In the upper half-plane there is infinite number of poles which are obtained from the equation,

$$
\frac{c}{a} e^{-a \tau / c}=-u+i \lambda
$$

which gives the pole locations

$$
\tau_{n}=-\frac{c}{a} \ln \left[\frac{a}{c}(u-i \lambda)\right]+\frac{\pi c(2 n-1)}{a} i,
$$

where $n=1,2, \ldots$ is a positive integer. As a result, we obtain

$$
F_{R}(t, z)=-\frac{\pi g}{\hbar}\left(-\frac{a}{c}\right)^{-i \omega c / a}(u-i \lambda)^{-i \omega c / a} \sum_{n=1}^{\infty} e^{-2 \pi c \omega n / a}=-\frac{\pi g}{\hbar}\left(-\frac{a}{c}\right)^{-i \omega c / a} \frac{(u-i \lambda)^{-i \omega c / a}}{e^{2 \pi c \omega / a}-1} .
$$

That is the right-propagating photon is emitted into the mode which has the form

$$
F_{R}(u)=N(u-i \lambda)^{-i(c \omega / a)},
$$


where $N$ is the normalization factor. It is known as the Unruh-Minkowski mode. The normalization factor is chosen such that the Unruh-Minkowski modes and the Rindler-modes (5) and (6) have the same normalization.

Similar calculations yield for the left-moving mode,

$$
F_{L}(v)=N(v-i \lambda)^{i(c \omega / a)},
$$

where

$$
v=t+z / c
$$

\section{APPENDIX C: COMMUTATOR OF THE ELECTRIC-FIELD OPERATORS IN ONE DIMENSION}

Using expression for the electric-field operator (21) in terms of plane-wave mode functions (22),

$$
\hat{E}(t, \mathbf{r})=-i \sum_{\mathbf{k}} \sqrt{\frac{v_{k}}{2}}\left(\hat{a}_{\mathbf{k}} e^{-i \nu_{k} t+i \mathbf{k} \cdot \mathbf{r}}-\hat{a}_{\mathbf{k}}^{\dagger} e^{i v_{k} t-i \mathbf{k} \cdot \mathbf{r}}\right),
$$

and $v_{k}=c k$, we obtain the following expression for the commutator of the electric-field operators in one dimension,

$$
\begin{aligned}
{\left[\hat{E}\left(t^{\prime}, z_{1}\right), \hat{E}\left(t^{\prime \prime}, z_{2}\right)\right] } & =\frac{1}{2} \sum_{\mathbf{k}} v_{k}\left(e^{-i v_{k}\left(t^{\prime}-t^{\prime \prime}\right)+i \mathbf{k} \cdot\left(\mathbf{z}_{1}-\mathbf{z}_{2}\right)}-\text { c.c. }\right) \\
& =\frac{c}{4 \pi} \int_{0}^{\infty} d k k\left(e^{-i c k\left(t^{\prime}-t^{\prime \prime}\right)+i k\left(z_{1}-z_{2}\right)}+e^{-i c k\left(t^{\prime}-t^{\prime \prime}\right)-i k\left(z_{1}-z_{2}\right)}-\text { c.c. }\right) \\
& =\frac{i c}{4 \pi} \frac{\partial}{\partial z_{2}} \int_{-\infty}^{\infty} d k\left(e^{-i c k\left(t^{\prime}-t^{\prime \prime}\right)+i k\left(z_{1}-z_{2}\right)}-e^{-i c k\left(t^{\prime}-t^{\prime \prime}\right)-i k\left(z_{1}-z_{2}\right)}\right),
\end{aligned}
$$

where we set the photon length to be equal to 1 . Taking into account that

$$
\int_{-\infty}^{\infty} d k e^{i k\left(z_{1}-z_{2}\right)}=2 \pi \delta\left(z_{1}-z_{2}\right)
$$

we find

$$
\left[\hat{E}\left(t^{\prime}, z_{1}\right), \hat{E}\left(t^{\prime \prime}, z_{2}\right)\right]=\frac{i c}{2} \frac{\partial}{\partial z_{2}}\left\{\delta\left[c\left(t^{\prime}-t^{\prime \prime}\right)+z_{2}-z_{1}\right]-\delta\left[c\left(t^{\prime}-t^{\prime \prime}\right)+z_{1}-z_{2}\right]\right\}
$$

\section{APPENDIX D: SIMULTANEOUS EXCITATION OF TWO ATOMS ACCELERATED IN THE OPPOSITE RINDLER WEDGES}

Here we consider two electrically neutral two-level ( $a$ and $b$ ) atoms with transition angular frequencies $\omega_{1}$ and $\omega_{2}$. We assume that atom 1 accelerates in the right Rindler wedge along the trajectory,

$$
t(\tau)=\frac{c}{a} \sinh \left(\frac{a \tau}{c}\right), \quad z_{1}(\tau)=\frac{c^{2}}{a} \cosh \left(\frac{a \tau}{c}\right),
$$

whereas atom 2 accelerates in the left Rindler wedge along the trajectory (see Fig. 5),

$$
t(\tau)=\frac{c}{a} \sinh \left(\frac{a \tau}{c}\right), \quad z_{2}(\tau)=-\frac{c^{2}}{a} \cosh \left(\frac{a \tau}{c}\right) .
$$

The acceleration of the atoms has the same magnitude but opposite sign.

The interaction Hamiltonian between the atoms and the field is

$$
\hat{V}(\tau)=\hat{V}_{1}(\tau)+\hat{V}_{2}(\tau)
$$

where

$$
\begin{aligned}
& \hat{V}_{1}(\tau)=g\left(\hat{\sigma}_{1} e^{-i \omega_{1} \tau}+\hat{\sigma}_{1}^{\dagger} e^{i \omega_{1} \tau}\right) \frac{\partial \hat{\Phi}\left[t(\tau), z_{1}(\tau)\right]}{\partial \tau}, \\
& \hat{V}_{2}(\tau)=g\left(\hat{\sigma}_{2} e^{-i \omega_{2} \tau}+\hat{\sigma}_{2}^{\dagger} e^{i \omega_{2} \tau}\right) \frac{\partial \hat{\Phi}\left[t(\tau), z_{2}(\tau)\right]}{\partial \tau},
\end{aligned}
$$

$\tau$ is the proper time of atoms 1 and 2, $\hat{\sigma}$ is the atomic lowering operator, and $g$ is the atom-field coupling constant. We write the field operator $\hat{\Phi}$ in terms of the right- and left-propagating Rindler modes,

$$
\hat{\Phi}(t, z)=\hat{\Phi}_{R}(t, z)+\hat{\Phi}_{L}(t, z)
$$


where the field operators for the right- $R$ and left- $L$ propagating modes in the Minkowski coordinates are given by

$$
\begin{aligned}
\hat{\Phi}_{R}(t, z)= & \int_{0}^{\infty} \frac{d v}{\sqrt{v}}\left[\hat{b}_{R 1 v} e^{i(v c / a) \ln \left[\left(a / c^{2}\right)(z-c t)\right]} \theta(z-c t)+\hat{b}_{R 2 v} e^{-i(v c / a) \ln \left[\left(a / c^{2}\right)(c t-z)\right]} \theta(c t-z)\right. \\
& \left.+\hat{b}_{R 1 v}^{\dagger} e^{-i(v c / a) \ln \left[\left(a / c^{2}\right)(z-c t)\right]} \theta(z-c t)+\hat{b}_{R 2 v}^{\dagger} e^{i(v c / a) \ln \left[\left(a / c^{2}\right)(c t-z)\right]} \theta(c t-z)\right], \\
\hat{\Phi}_{L}(t, z)= & \int_{0}^{\infty} \frac{d v}{\sqrt{v}}\left[\hat{b}_{L 2 v} e^{-i(v c / a) \ln \left[\left(a / c^{2}\right)(z+c t)\right]} \theta(z+c t)+\hat{b}_{L 1 \nu} e^{i(v c / a) \ln \left[\left(a / c^{2}\right)(-z-c t)\right]} \theta(-c t-z)\right. \\
& \left.+\hat{b}_{L 2 v}^{\dagger} e^{i(v c / a) \ln \left[\left(a / c^{2}\right)(z+c t)\right]} \theta(z+c t)+\hat{b}_{L 1 v}^{\dagger} e^{-i(v c / a) \ln \left[\left(a / c^{2}\right)(-z-c t)\right]} \theta(-c t-z)\right],
\end{aligned}
$$

$\hat{b}_{v}$ and $\hat{b}_{v}^{\dagger}$ are annihilation and creation operators of the Rindler photons. Along the atomic trajectories we have

$$
\begin{aligned}
& z_{1}(\tau)-c t(\tau)=\frac{c^{2}}{a} e^{-(a \tau / c)}, \quad z_{1}(\tau)+c t(\tau)=\frac{c^{2}}{a} e^{(a \tau / c)}, \\
& z_{2}(\tau)-c t(\tau)=-\frac{c^{2}}{a} e^{(a \tau / c)}, \quad z_{2}(\tau)+c t(\tau)=-\frac{c^{2}}{a} e^{-(a \tau / c)} .
\end{aligned}
$$

Therefore, along the atomic trajectories,

$$
\begin{aligned}
& \hat{\Phi}_{R}\left[t(\tau), z_{1}(\tau)\right]=\int_{0}^{\infty} \frac{d \nu}{\sqrt{\nu}}\left(\hat{b}_{R 1 \nu} e^{-i \nu \tau}+\hat{b}_{R 1 \nu}^{\dagger} e^{i \nu \tau}\right), \\
& \hat{\Phi}_{L}\left[t(\tau), z_{1}(\tau)\right]=\int_{0}^{\infty} \frac{d v}{\sqrt{\nu}}\left(\hat{b}_{L 2 v} e^{-i \nu \tau}+\hat{b}_{L 2 \nu}^{\dagger} e^{i \nu \tau}\right), \\
& \hat{\Phi}_{R}\left[t(\tau), z_{2}(\tau)\right]=\int_{0}^{\infty} \frac{d \nu}{\sqrt{\nu}}\left(\hat{b}_{R 2 \nu} e^{-i \nu \tau}+\hat{b}_{R 2 \nu}^{\dagger} e^{i \nu \tau}\right), \\
& \hat{\Phi}_{L}\left[t(\tau), z_{2}(\tau)\right]=\int_{0}^{\infty} \frac{d \nu}{\sqrt{v}}\left(\hat{b}_{L 1 \nu} e^{-i \nu \tau}+\hat{b}_{L 1 \nu}^{\dagger} e^{i \nu \tau}\right) .
\end{aligned}
$$

We assume that initially (at $t_{0}=-\infty$ ) both atoms are in the ground-state $b$ and the field is in Minkowski vacuum $\left|0_{M}\right\rangle$. That is, the initial-state vector of the system is

$$
\left|\psi_{0}\right\rangle=\left|b_{1} b_{2} 0_{M}\right\rangle
$$

We will use representation (26) for the evolution operator which is valid in the second order in the interaction $\hat{V}$. We are interested in the probability that at $t=+\infty$ both atoms become excited and the field remains in Minkowski vacuum state $\left|0_{M}\right\rangle$. Since atoms 1 and 2 move in the causally disconnected regions the second term on the right-hand side of Eq. (26) yields no contribution.

In the first term one can take

$$
\hat{U}_{1,2}(t) \approx 1-\frac{i}{\hbar} \int_{t_{0}}^{t} d t^{\prime} \hat{V}_{1,2}\left(t^{\prime}\right)
$$

Thus, the probability that both atoms become excited and the field is in Minkowski vacuum state is given by

$$
P_{a_{1} a_{2} 0}=\frac{1}{\hbar^{4}}\left|\left\langle a_{1} a_{2} 0_{M}\left|\int_{-\infty}^{\infty} d \tau^{\prime} \hat{V}_{1}\left(\tau^{\prime}\right) \int_{-\infty}^{\infty} d \tau^{\prime \prime} \hat{V}_{2}\left(\tau^{\prime \prime}\right)\right| b_{1} b_{2} 0_{M}\right\rangle\right|^{2} .
$$

Plugging $\hat{V}_{1}(\tau)$ and $\hat{V}_{2}(\tau)$, and taking into account that

$$
\begin{gathered}
\int_{-\infty}^{\infty} d \tau^{\prime} e^{i \omega_{1} \tau^{\prime}} \frac{\partial \hat{\Phi}\left[\tau^{\prime}, z_{1}\left(\tau^{\prime}\right)\right]}{\partial \tau^{\prime}}=-2 \pi i \sqrt{\omega_{1}}\left(\hat{b}_{R 1 \omega_{1}}+\hat{b}_{L 2 \omega_{1}}\right), \\
\int_{-\infty}^{\infty} d \tau^{\prime \prime} e^{i \omega_{2} \tau^{\prime \prime}} \frac{\partial \hat{\Phi}\left[\tau^{\prime \prime}, z_{2}\left(\tau^{\prime \prime}\right)\right]}{\partial \tau^{\prime \prime}}=-2 \pi i \sqrt{\omega_{2}}\left(\hat{b}_{R 2 \omega_{2}}+\hat{b}_{L 1 \omega_{2}}\right),
\end{gathered}
$$

we obtain

$$
P_{a_{1} a_{2} 0}=\frac{16 \pi^{4} g^{4} \omega_{1} \omega_{2}}{\hbar^{4}}\left|\left\langle 0_{M}\left|\left(\hat{b}_{R 1 \omega_{1}} \hat{b}_{R 2 \omega_{2}}+\hat{b}_{L 2 \omega_{1}} \hat{b}_{L 1 \omega_{2}}\right)\right| 0_{M}\right\rangle\right|^{2} .
$$

One can calculate the matrix element using relations (14) between operators for the Rindler-modes $\hat{b}_{v}$ and the UnruhMinkowski modes $\hat{a}_{v}[18]$,

$$
\begin{aligned}
\hat{b}_{R 1 v} & =\frac{\hat{a}_{R 1 v}+e^{-\pi c v / a} \hat{a}_{R 2 v}^{\dagger}}{\sqrt{1-e^{-2 \pi c v / a}}}, \quad \hat{b}_{R 2 v}=\frac{\hat{a}_{R 2 v}+e^{-\pi c v / a} \hat{a}_{R 1 v}^{\dagger}}{\sqrt{1-e^{-2 \pi c v / a}}}, \\
\hat{b}_{L 1 v} & =\frac{\hat{a}_{L 1 v}+e^{-\pi c v / a} \hat{a}_{L 2 v}^{\dagger}}{\sqrt{1-e^{-2 \pi c v / a}}}, \quad \hat{b}_{L 2 v}=\frac{\hat{a}_{L 2 v}+e^{-\pi c v / a} \hat{a}_{L 1 v}^{\dagger}}{\sqrt{1-e^{-2 \pi c v / a}}} .
\end{aligned}
$$


As a result, we obtain

$$
\left\langle 0_{M}\left|\left(\hat{b}_{R 1 \omega_{1}} \hat{b}_{R 2 \omega_{2}}+\hat{b}_{L 2 \omega_{1}} \hat{b}_{L 1 \omega_{2}}\right)\right| 0_{M}\right\rangle=\frac{\delta_{\omega_{1} \omega_{2}}}{\sinh \left(\pi c \omega_{1} / a\right)}
$$

and, therefore,

$$
P_{a_{1} a_{2} 0}=\frac{16 \pi^{4} g^{4} \omega_{1} \omega_{2} \delta_{\omega_{1} \omega_{2}}}{\hbar^{4} \sinh ^{2}\left(\pi c \omega_{1} / a\right)} .
$$

On the other hand, the probability that atom 1 emits a photon into the right-moving Unruh-Minkowski mode with frequency $\omega_{1}$ and becomes excited is given by

$$
P_{a_{1} R}=\frac{g^{2}}{\hbar^{2}}\left|\left\langle 1_{U M \omega_{1} R}\left|\int_{-\infty}^{\infty} d \tau^{\prime} e^{i \omega_{1} \tau^{\prime}} \frac{\partial \hat{\Phi}\left[\tau^{\prime}, z_{1}\left(\tau^{\prime}\right)\right]}{\partial \tau^{\prime}}\right| 0_{M}\right\rangle\right|^{2}=\frac{2 \pi^{2} g^{2} \omega_{1} e^{-\pi c \omega_{1} / a}}{\hbar^{2} \sinh \left(\pi c \omega_{1} / a\right)} .
$$

The probability of photon emission into the left-moving Unruh-Minkowski mode is given by the same expression. Thus, we find

$$
P_{a_{2}}=\frac{4 \pi^{2} g^{2} \omega_{1} e^{-\pi c \omega_{1} / a}}{\hbar^{2} \sinh \left(\pi c \omega_{1} / a\right)} .
$$

Similarly, for the excitation probability of atom 2 we obtain

$$
P_{a_{2}}=P_{a_{2} R}+P_{a_{2} L}=\frac{4 \pi^{2} g^{2} \omega_{2} e^{-\pi c \omega_{2} / a}}{\hbar^{2} \sinh \left(\pi c \omega_{2} / a\right)}
$$

Comparing this with Eq. (D6) we find that for $\omega_{1}=\omega_{2}$,

$$
P_{a_{1} a_{2} 0}=e^{2 \pi c \omega_{1} / a} P_{a_{1}} P_{a_{2}}
$$

That is, $P_{a_{1} a_{2} 0}>P_{a_{1}} P_{a_{2}}$.

\section{APPENDIX E: BOGOLIUBOV TRANSFORMATION AND VACUUM STATE}

Consider the following Bogoliubov transformation:

$$
\hat{a}=\alpha \hat{b}-\beta \hat{c}^{\dagger},
$$

where $\hat{a}, \hat{b}$, and $\hat{c}^{\dagger}$ are annihilation and creation operators of photons in modes $a$, $b$, and $c$; operator $\hat{c}$ commutes with $\hat{b}$ and $\left[\hat{b}, \hat{b}^{\dagger}\right]=1$.

We denote as $\left|0_{a}\right\rangle$ and $\left|0_{b}\right\rangle$ vacuum states for the operators $\hat{a}$ and $\hat{b}$, respectively, that is $\hat{a}\left|0_{a}\right\rangle=0$ and $\hat{b}\left|0_{b}\right\rangle=0$. Here we show that relation between $\left|0_{a}\right\rangle$ and $\left|0_{b}\right\rangle$ is

$$
\left|0_{a}\right\rangle=N e^{(\beta / \alpha) \hat{b}^{\dagger} \hat{c}^{\dagger}}\left|0_{b}\right\rangle=N \sum_{n=0}^{\infty}\left(\frac{\beta}{\alpha}\right)^{n}|n, n\rangle,
$$

where $N$ is a normalization factor and $|n, n\rangle$ is a state with $n$ photons in modes $b$ and $c$. The value of $N$ is fixed by normalization $\left\langle 0_{a} \mid 0_{a}\right\rangle=1$ which yields

$$
N=\frac{1}{\sqrt{\sum_{n=0}^{\infty}\left(\frac{\beta}{\alpha}\right)^{2 n}}}=\sqrt{1-\left(\frac{\beta}{\alpha}\right)^{2}} .
$$

Using the identity,

$$
\hat{b} e^{(\beta / \alpha) \hat{b}^{\dagger} \hat{c}^{\dagger}}=e^{(\beta / \alpha) \hat{b}^{\dagger} \hat{c}^{\dagger}}\left(\hat{b}+\frac{\beta}{\alpha} \hat{c}^{\dagger}\right),
$$

we obtain

$$
\hat{a}\left|0_{a}\right\rangle=\left(\alpha \hat{b}-\beta \hat{c}^{\dagger}\right)\left|0_{a}\right\rangle=N\left(\alpha \hat{b}-\beta \hat{c}^{\dagger}\right) e^{(\beta / \alpha) \hat{b}^{\dagger} \hat{c}^{\dagger}}\left|0_{b}\right\rangle=\alpha N e^{(\beta / \alpha) \hat{b}^{\dagger} \hat{c}^{\dagger}} \hat{b}\left|0_{b}\right\rangle=0 .
$$

That is, state (E1) is the vacuum state for the operator $\hat{a}$ provided $\left|0_{b}\right\rangle$ is the vacuum state for operator $\hat{b}$.

To prove the identity (E2), or

$$
e^{-\gamma \hat{b}^{\dagger} \hat{c}^{\dagger}} \hat{b} e^{\gamma \hat{b}^{\dagger} \hat{c}^{\dagger}}=\hat{b}+\gamma \hat{c}^{\dagger}
$$

where $\gamma=\beta / \alpha$, we consider an operator,

$$
\hat{A}(\gamma)=e^{-\gamma \hat{b}^{\dagger} \hat{c}^{\dagger}} \hat{b} e^{\gamma \hat{b}^{\dagger} \hat{c}^{\dagger}}
$$


Operator $\hat{A}(\gamma)$ obeys the differential equation,

$$
\frac{d \hat{A}}{d \gamma}=\hat{c}^{\dagger} e^{-\gamma \hat{b}^{\dagger} \hat{c}^{\dagger}}\left[\hat{b}, \hat{b}^{\dagger}\right] e^{\gamma^{\dagger} \hat{c}^{\dagger}}=\hat{c}^{\dagger}
$$

subject to the initial condition $\hat{A}(\gamma=0)=\hat{b}$. The solution of the differential equation is $\hat{A}=\hat{b}+\gamma \hat{c}^{\dagger}$.

\section{APPENDIX F: $\delta$-FUNCTION DETECTOR}

Here we consider a pair of two-level $\left(a\right.$ and $b$ ) atoms with transition angular frequencies $\omega_{1}$ and $\omega_{2}$. We assume that atom 2 is fixed at a coordinate $z_{2}$ and the other atom is moving along the $z$ axis. Atom 2 serves as a detector which is suddenly switched on and off at time $t=T$. We will model this process as a $\delta$-function coupling in the interaction Hamiltonian between atom 2 and the field.

We are interested in the probability that both atoms become excited as a function of the position of detector atom $z_{2}$ and time $T$. The interaction Hamiltonian between the atoms and the field is given by Eqs. (18)-(21) in which $f(t)=\delta(t-T)$. We assume that at the initial moment of time $t_{0}<T$ both atoms are in the ground-state $b$ and the field is in the Minkowski vacuum $\left|0_{M}\right\rangle$. That is, the initial-state vector of the system is

$$
\left|\psi\left(t_{0}\right)\right\rangle=\left|b_{1} b_{2} 0\right\rangle .
$$

If the interaction is weak, the state vector of the system at time $t$ can be found using the perturbation theory. In the second order, we obtain

$$
|\psi(t)\rangle \approx\left|\psi\left(t_{0}\right)\right\rangle-\frac{i}{\hbar} \int_{t_{0}}^{t} d t^{\prime} \hat{V}\left(t^{\prime}\right)\left|\psi\left(t_{0}\right)\right\rangle-\frac{1}{\hbar^{2}} \int_{t_{0}}^{t} d t^{\prime} \int_{t_{0}}^{t^{\prime}} d t^{\prime \prime} \hat{V}\left(t^{\prime}\right) \hat{V}\left(t^{\prime \prime}\right)\left|\psi\left(t_{0}\right)\right\rangle
$$

The contribution to the probability amplitude that both atoms become excited and the field is in Minkowski vacuum $\left\langle a_{1} a_{2} 0 \mid \psi(t)\right\rangle$ come from the cross terms. Plugging Eqs. (F1) and (18) into the probability amplitude gives

$$
\left\langle a_{1} a_{2} 0 \mid \psi(t)\right\rangle \approx-\frac{1}{\hbar^{2}}\left\langle a_{1} a_{2} 0\left|\int_{t_{0}}^{t} d t^{\prime} \int_{t_{0}}^{t^{\prime}} d t^{\prime \prime}\left[\hat{V}_{1}\left(t^{\prime}\right) \hat{V}_{2}\left(t^{\prime \prime}\right)+\hat{V}_{2}\left(t^{\prime}\right) \hat{V}_{1}\left(t^{\prime \prime}\right)\right]\right| b_{1} b_{2} 0\right\rangle .
$$

Taking into account Eqs. (19) and (20) we obtain for $t \geqslant T$,

$$
\left\langle a_{1} a_{2} 0 \mid \psi(t)\right\rangle \approx-\frac{g^{2}}{\hbar^{2}}\left\langle 0\left|\int_{T}^{t} d t^{\prime} e^{i \omega_{1} t^{\prime}+i \omega_{2} T} \hat{E}\left[t^{\prime}, \mathbf{r}_{1}\left(t^{\prime}\right)\right] \hat{E}\left(T, \mathbf{r}_{2}\right)\right| 0\right\rangle-\frac{g^{2}}{\hbar^{2}}\left\langle 0\left|\int_{t_{0}}^{T} d t^{\prime \prime} e^{i \omega_{1} t^{\prime \prime}+i \omega_{2} T} \hat{E}\left(T, \mathbf{r}_{2}\right) \hat{E}\left[t^{\prime \prime}, \mathbf{r}_{1}\left(t^{\prime \prime}\right)\right]\right| 0\right\rangle
$$

One can disregard the first term on the right-hand side of Eq. (F2) if $t=T$, that is, if we calculate the excitation probability at the moment of time when the $\delta$-function detector makes the measurement. Plugging Eqs. (21) and (22) gives for $t=T$,

$$
\left\langle a_{1} a_{2} 0 \mid \psi(T)\right\rangle \approx-\frac{g^{2}}{2 \hbar^{2}} \sum_{\mathbf{k}} v_{k} \int_{t_{0}}^{T} d t^{\prime} e^{i \omega_{1} t^{\prime}+i \omega_{2} T} e^{i \nu_{k} t^{\prime}-i \mathbf{k} \cdot \mathbf{r}_{1}\left(t^{\prime}\right)} e^{-i v_{k} T+i \mathbf{k} \cdot \mathbf{r}_{2}} .
$$

For the one-dimensional problem replacing the sum over $\mathbf{k}$ by an integral,

$$
\sum_{\mathbf{k}} \rightarrow \frac{1}{2 \pi} \int_{-\infty}^{\infty} d k
$$

where we set the photon quantization length to be equal to 1 , we obtain

$$
\left\langle a_{1} a_{2} 0 \mid \psi(T)\right\rangle \approx-\frac{c g^{2}}{4 \pi \hbar^{2}} \int_{-\infty}^{\infty} d k|k| \int_{-\infty}^{T} d t^{\prime} e^{i \omega_{1} t^{\prime}+i \omega_{2} T} e^{i c|k| t^{\prime}-i k z_{1}\left(t^{\prime}\right)} e^{-i c|k| T+i k z_{2}}
$$

Since atom 1 is accelerated, the integration over $t^{\prime}$ should be replaced with the integration over the atom's proper time $\tau$. Separating contributions from the right- and the left-propagating modes one can write the probability amplitude as

$$
\begin{aligned}
\left\langle a_{1} a_{2} 0 \mid \psi(T)\right\rangle \approx & -\frac{g^{2}}{4 \pi c \hbar^{2}} e^{i \omega_{2} T}\left(\int_{0}^{\infty} d \nu v e^{-i \nu\left(T-z_{2} / c\right)} \int_{-\infty}^{T} d \tau e^{i v\left[t(\tau)-z_{1}(\tau) / c\right]} e^{i \omega_{1} \tau}\right. \\
& \left.+\int_{0}^{\infty} d v v e^{-i v\left(T+z_{2} / c\right)} \int_{-\infty}^{T} d \tau e^{i v\left[t(\tau)+z_{1}(\tau) / c\right]} e^{i \omega_{1} \tau}\right) .
\end{aligned}
$$

We calculate the integrals in Eq. (F3) for a uniformly accelerated atom 1. The atom's trajectory is given by Eq. (10). We assume that coupling between the field and atom 1 is switched on and off adiabatically before the measurement time $T$. Then expressions under the integral over $d \tau$ in Eq. (F3) must be multiplied by a function $g(\tau)$ which is equal to 1 when the interaction is on and zero otherwise. 
Next we calculate the integral describing the contribution from the right-propagating photons,

$$
I_{R}=\int_{-\infty}^{T} d \tau g(\tau) e^{i \nu\left[t(\tau)-z_{1}(\tau) / c\right]} e^{i \omega_{1} \tau}=\int_{-\infty}^{T} d \tau g(\tau) e^{i \phi(\tau)},
$$

where

$$
\phi(\tau)=v t(\tau)-v z_{1}(\tau) / c+\omega_{1} \tau
$$

Integrating by parts, one can write $I_{R}$ as

$$
I_{R}=\left.\frac{g(\tau) e^{i \phi(\tau)}}{i \frac{\partial \phi}{\partial \tau}}\right|_{-\infty} ^{T}-\int_{-\infty}^{T} d \tau \frac{\partial g(\tau)}{\partial \tau} \frac{e^{i \phi(\tau)}}{i \frac{\partial \phi}{\partial \tau}}+\int_{-\infty}^{T} d \tau \frac{\partial^{2} \phi}{\partial \tau^{2}} \frac{g(\tau) e^{i \phi(\tau)}}{i\left(\frac{\partial \phi}{\partial \tau}\right)^{2}} .
$$

If the interaction is switched on and off adiabatically, one can disregard the first two terms on the right-hand side of Eq. (F4), and in the last term extend the integration over $\tau$ to $+\infty$. Atomic excitation can occur only from a nonadiabatic change in $\partial \phi / \partial \tau$. Inserting Eq. (10) into $\phi(\tau)$ we obtain

$$
\phi=-\frac{\nu c}{a} e^{-a \tau / c}+\omega_{1} \tau, \quad \frac{\partial \phi}{\partial \tau}=\omega_{1}+\nu e^{-a \tau / c}, \quad \frac{\partial^{2} \phi}{\partial \tau^{2}}=-\frac{a \nu}{c} e^{-a \tau / c},
$$

and, therefore,

$$
I_{R}=\frac{i a \nu}{c} \int_{-\infty}^{\infty} d \tau \frac{g(\tau) e^{-i(v c / a) e^{-a \tau / c}} e^{i \omega_{1} \tau}}{\left(\nu e^{-a \tau / 2 c}+\omega_{1} e^{a \tau / 2 c}\right)^{2}}
$$

The contribution to the integral comes from the region $-c / a \lesssim \tau \lesssim c / a$. Assuming that $g(\tau)=1$ in this region one can take $g(\tau)$ out of the integral. Then, performing the change in the integration variable to $x=\frac{\nu}{\omega_{1}} e^{-a \tau / c}$ yields

$$
I_{R}=\frac{i}{\omega_{1}}\left(\frac{v}{\omega_{1}}\right)^{\left(i c \omega_{1}\right) / a} \int_{0}^{\infty} d x \frac{e^{-i\left(c \omega_{1} x\right) / a} x^{-\left(i c \omega_{1}\right) / a}}{(x+1)^{2}} .
$$

The dependence on frequency $v$ is only present in the factor $v^{\left(i c \omega_{1}\right) / a}$. The integral over $x$ can be expressed in terms of the $\gamma$ function. Namely,

$$
\int_{0}^{\infty} d x \frac{e^{-i\left(c \omega_{1} x\right) / a} x^{-\left(i c \omega_{1} / a\right)}}{(x+1)^{2}}=-i\left(\frac{i c \omega_{1}}{a}\right)^{\left(i c \omega_{1}\right) / a} \frac{c \omega_{1}}{a} \Gamma\left(-\frac{i c \omega_{1}}{a}\right)
$$

and, therefore,

$$
I_{R}=\frac{c}{a}\left(\frac{i \nu c}{a}\right)^{\left(i c \omega_{1}\right) / a} \Gamma\left(-\frac{i c \omega_{1}}{a}\right)
$$

Next we calculate the integral over $v$ and obtain

$$
\int_{0}^{\infty} d v v e^{-i v\left(T-z_{2} / c\right)} v^{\left(i c \omega_{1}\right) / a}=\frac{-\pi i}{\sinh \left(\frac{\pi c \omega_{1}}{a}\right)} \frac{1+\frac{i c \omega_{1}}{a}}{\Gamma\left(-\frac{i c \omega_{1}}{a}\right)\left|z_{2} / c-T\right|^{2+i c \omega_{1} / a}} \begin{cases}e^{-\left(\pi c \omega_{1}\right) / 2 a}, & \frac{z_{2}}{c}-T>0 \\ e^{\left(\pi c \omega_{1}\right) / 2 a}, & T-\frac{z_{2}}{c}>0 .\end{cases}
$$

As a result, for the right-propagating modes,

$$
\int_{0}^{\infty} d \nu v e^{-i \nu\left(T-z_{2} / c\right)} \int_{-\infty}^{T} d \tau e^{i v\left[t(\tau)-z_{1}(\tau) / c\right]} e^{i \omega_{1} \tau}=\frac{-\pi\left(\frac{i c}{a}\right)^{\left[\left(i c \omega_{1}\right) / a\right]+1}}{\sinh \left(\frac{\pi c \omega_{1}}{a}\right)} \frac{1+\frac{i c \omega_{1}}{a}}{\left|z_{2} / c-T\right|^{2+\frac{i c \omega_{1}}{a}}} \begin{cases}e^{-\left(\pi c \omega_{1}\right) / 2 a}, & \frac{z_{2}}{c}-T>0 \\ e^{\left(\pi c \omega_{1}\right) / 2 a}, & T-\frac{z_{2}}{c}>0 .\end{cases}
$$

If we disregard the left-propagating modes [the second term in Eq. (F3)] then the probability that both atoms are excited at time $T$ and the field is in Minkowski vacuum is

$$
P_{a_{2} a_{1} 0}\left(T, z_{2}\right)=\frac{g^{4}}{16 \hbar^{4} a^{2}} \frac{1+\left(\frac{c \omega_{1}}{a}\right)^{2}}{\sinh ^{2}\left(\frac{\pi c \omega_{1}}{a}\right)} \frac{e^{-\left[\left(2 \pi c \omega_{1}\right) / a\right] \theta\left(z_{2} / c-T\right)}}{\left(z_{2} / c-T\right)^{4}},
$$

where we used $\left|i^{i \alpha}\right|^{2}=e^{-\pi \alpha}$. Equation (F5) shows that the conditional probability $P_{a_{2} a_{1} 0}\left(T, z_{2}\right)$ is in the factor exp $\left(2 \pi c \omega_{1} / a\right)$ larger in the region $z_{2} / c-T<0$, which is causally disconnected from the accelerated atom 1 .

[1] M. I. Shirokov, Signal velocity in quantum electrodynamics, Sov. Phys. Usp. 21, 345 (1978).
[2] T. D. Lee and G. C. Wick, Finite theory of quantum electrodynamics, Phys. Rev. D 2, 1033 (1970). 
[3] G. C. Hegerfeldt, Remark on causality and particle localization, Phys. Rev. D 10, 3320 (1974).

[4] J. Fernando Perez and I. F. Wilde, Localization and causality in relativistic quantum mechanics, Phys. Rev. D 16, 315 (1977).

[5] A. K. Biswas, G. Compagno, G. M. Palma, R. Passante and F. Persico, Virtual photons and causality in the dynamics of a pair of two-level atoms, Phys. Rev. A 42, 4291 (1990).

[6] G. C. Hegerfeldt, Instantaneous spreading and Einstein causality in quantum theory, Ann. Phys. (Leipzig) 7, 716 (1998).

[7] V. P. Bykov and V. I. Tatarskii, Causality violation in the Glauber theory of photodetection, Phys. Lett. A 136, 77 (1989).

[8] V. I. Tatarskii, Corrections to the theory of photocounting, Phys. Lett. A 144, 491 (1990).

[9] M. de Haan, Photodetection and causality I, Physica A 132, 375 (1985).

[10] M. de Haan, Photodetection and causality II, Physica A 132, 397 (1985).

[11] E. Fermi, Quantum theory of radiation, Rev. Mod. Phys. 4, 87 (1932).

[12] P. W. Milonni, D. F. V. James, and H. Fearn, Photodetection and causality in quantum optics, Phys. Rev. A 52, 1525 (1995).

[13] L. I. Plimak, S. T. Stenholm, and W. P. Schleich, Operator ordering and causality, Phys. Scr., T 147, 014026 (2012).

[14] W. G. Unruh, Notes on black hole evaporation, Phys. Rev. D. 14, 870 (1976).

[15] S. A. Fulling, Nonuniqueness of canonical field quantization in riemannian space time, Phys. Rev. D 7, 2850 (1973).

[16] P. C. W. Davies, Scalar production in Schwarzschild and Rindler metrics, J. Phys. A 8, 609 (1975).

[17] W. Rindler, Kruskal space and the uniformly accelerated frame, Am. J. Phys. 34, 1174 (1966).

[18] W. G. Unruh and R. M. Wald, What happens when an accelerating observer detects a Rindler particle, Phys. Rev. D 29, 1047 (1984).
[19] M. O. Scully, S. Fulling, D. M. Lee, D. N. Page, W. P. Schleich, and A. A. Svidzinsky, Quantum optics approach to radiation from atoms falling into a black hole, Proc. Natl. Acad. Sci. USA 115, 8131 (2018).

[20] A. A. Svidzinsky, Excitation of a uniformly moving atom through vacuum fluctuations, Phys. Rev. Res. 1, 033027 (2019).

[21] A. A. Svidzinsky, J. S. Ben-Benjamin, S. A. Fulling, and D. N. Page, Excitation of an Atom by a Uniformly Accelerated Mirror through Virtual Transitions, Phys. Rev. Lett. 121, 071301 (2018).

[22] M. O. Scully, A. A. Svidzinsky, and W. Unruh, Causality in acceleration radiation, Phys. Rev. Res. 1, 033115 (2019).

[23] A. A. Svidzinsky, A. Azizi, J. S. Ben-Benjamin, M. O. Scully, and W. Unruh, Unruh and Cherenkov Radiation from a Negative Frequency Perspective, Phys. Rev. Lett. 126, 063603 (2021).

[24] A. Pozas-Kerstjens and E. Martín-Martínez, Harvesting correlations from the quantum vacuum, Phys. Rev. D 92, 064042 (2015).

[25] B. Reznik, Distillation of vacuum entanglement to EPR pairs, arXiv:quant-ph/0008006.

[26] B. Reznik, Entanglement from the Vacuum, Found. Phys. 33, 167 (2003).

[27] B. Reznik, A. Retzker, and J. Silman, Violating Bell's inequalities in vacuum, Phys. Rev. A 71, 042104 (2005).

[28] S. J. Olson and T. C. Ralph, Entanglement between the Future and the Past in the Quantum Vacuum, Phys. Rev. Lett. 106, 110404 (2011).

[29] S. J. Olson and T. C. Ralph, Extraction of timelike entanglement from the quantum vacuum, Phys. Rev. A 85, 012306 (2012).

[30] G. Salton, R. B. Mann, and N. C. Menicucci, Accelerationassisted entanglement harvesting and rangefinding, New J. Phys. 17, 035001 (2015). 